ESAIM: M2AN 49 (2015) 921-951

DOI: $10.1051 / \mathrm{m} 2 \mathrm{an} / 2014060$
ESAIM: Mathematical Modelling and Numerical Analysis

www.esaim-m2an.org

\title{
SHAPE OPTIMIZATION FOR STOKES FLOWS: A FINITE ELEMENT CONVERGENCE ANALYSIS *
}

\author{
Ivan Fumagalli $^{1}$, Nicola PAROlini ${ }^{1}$ AND Marco VERAni ${ }^{1}$
}

\begin{abstract}
In this paper we analyze a two-dimensional shape optimization problem, governed by Stokes equations that are defined on a domain with a part of the boundary that is described as the graph of the control function. The state problem formulation is mapped onto a reference domain, which is independent of the control function, and the analysis is mainly led on such domain. The existence of an optimal control function is proved, and optimality conditions are derived. After the analytical inspection of the problem, finite element discretization is considered for both the control function and the state variables, and a priori convergence error estimates are derived. Numerical experiments assess the validity of the theoretical results.
\end{abstract}

Mathematics Subject Classification. 49M25, 49Q10, 65N15, 65N30.

Received April 9, 2014. Revised December 6, 2014.

Published online May 20, 2015.

\section{INTRODUCTION}

Optimal control for partial differential equations [28] is a challenging field of applied mathematics, thanks to its combination of sophisticated theoretical tools and interesting engineering applications. Among optimal control problems, shape optimization $[12,22-24,33,35]$ has recently undergone a renewal of interest, mainly due to the wide range of industrial and real world applications, like fluid dynamics [19] and structural mechanics [1], and to the increased computational power available for numerical simulations. Shape optimization aims at finding the solution to problems of the following general form:

$$
\min _{\Omega \in \mathcal{O}} J(\Omega, S(\Omega)) \text {, subject to a differential problem } L(S(\Omega))=0 \text { in } \Omega,
$$

where $J$ is a cost functional, defined on a suitable set $\mathcal{O}$ of admissible domains, $L$ is a differential operator and $S$ is the operator mapping an admissible domain $\Omega \in \mathcal{O}$ to the corresponding solution of the differential problem $L(S(\Omega))=0$ in $\Omega$. In particular, in this paper we analyze a two-dimensional, steady Stokes problem, completed by mixed boundary conditions.

Problems of the form (1.1) have been widely discussed in the literature, employing different techniques in the description of the set $\mathcal{O}$, generally considered as a proper subset of finite (see, e.g., $[3,5]$ ) or infinite

\footnotetext{
Keywords and phrases. Shape optimization, Stokes problem, reference domain, convergence rates, finite elements.

* The third author has been partially supported by the Italian research grant Prin 2012 2012HBLYE4-004 "Metodologie innovative nella modellistica differenziale numerica".

1 MOX - Dipartimento di Matematica, Politecnico di Milano, P.zza Leonardo Da Vinci 32, 20133 Milano, Italy.

ivan.fumagalli@polimi.it; nicola.parolini@polimi.it; marco.verani@polimi.it
} 
(see, e.g., $[12,33,35])$ dimensional spaces. The present paper belongs to the latter category, as the boundary of the admissible domains (or a subset of it) is described by the graph of a suitable control function. This approach has been widely adopted by many authors (see, e.g., $[2,4,15,20,22,23,26,27]$ ).

Concerning the numerical solution of shape optimization problems, a standard technique is represented by gradient type iterative algorithms, in which the state problem is solved on differently shaped domains at each iteration (see, e.g., $[1,13]$ ). A critical point of this approach is the repeated deformation of the computational mesh, leading to an increase in the computational effort and to the possible generation of highly skewed mesh elements. In order to avoid such problems, a possible solution is to employ the so called reference domain approach (see, e.g., $[26,27]$ ) where, exploiting a suitable transformation, the actual domain and the whole optimization problem is mapped onto a reference domain $\Omega_{0}$, whose computational mesh is built once and for all. In this paper, we adopt the reference domain approach to study a shape optimization problem governed by Stokes equations. In particular, we will be interested in deriving a priori estimates for the different discretization errors (on state solution, control function and cost functional) involved in the finite element solution of the optimization problem. Discretization of shape optimization problems and convergence issues have been firstly addressed in $[9,10]$ and recently in $[16,26]$. To the best of our knowledge, $[16,26]$ are the only works providing, for a shape optimization problem governed by Poisson equations, a convergence rate for the discretization errors. On the other hand, analogous convergence results dealing with Stokes equations seem not to be available in the literature: inspired by [26] and extending the results therein contained, the present paper aims at filling this gap, by deriving novel finite element a priori error estimates and convergence rates for a shape optimization problem governed by Stokes equations.

It is worth remarking that, as one of the main goal of the paper is the proof of a priori error estimates for the FEM discretization errors, some more restrictive assumptions (w.r.t. the more general framework presented, e.g., in [33]) have to be employed. Indeed, these assumptions are instrumental to extract, via the application of interpolation error estimates, the correct convergence rates of the FEM errors.

Finally, we remark that considering Stokes equations is relevant for fluid-dynamics and elasticity applications. Moreover, it represents a first step towards the application of the reference domain approach to real world applications modeled by Navier-Stokes equations.

The paper is organized as follows. In Section 2, we present the shape optimization problem governed by Stokes equations, and we reformulate it on the reference domain. Within this framework, the existence of an optimal solution to the minimization problem is proved. Section 3 is devoted to the proof of a priori estimates for the numerical discretization error of the optimization problem. In Section 4, we consider first order optimality conditions and we provide a boundary-integral expression for them. Finally, in Section 5 we present some numerical tests, assessing the theoretical results. In Appendix A, we discuss the regularity assumptions needed by the a priori estimates, whereas in Appendix B some technical results are proved.

\section{The optimal CONTROL PROBlem}

The aim of the present paper is to study a shape optimization problem governed by Stokes equations, which reads as follows

$$
\begin{aligned}
& \min _{q \in Q^{a d}} J(q, \mathbf{u}, p) \text { subject to the following generalized Stokes system: } \\
& \left\{\begin{aligned}
\eta \mathbf{u}-\operatorname{div}(\nu \nabla \mathbf{u})+\nabla p & =\mathbf{f}, & & \text { in } \Omega_{q}, \\
\operatorname{div} \mathbf{u} & =0, & & \text { in } \Omega_{q}, \\
\mathbf{u} & =0, & & \text { on } \Gamma_{q}, \\
\nu \partial_{\mathbf{n}} \mathbf{u}-p \mathbf{n} & =\mathbf{g}_{N}, & & \text { on } \Gamma_{1}, \\
\partial_{\mathbf{n}} u_{x}=0, \quad u_{y} & =0, & & \text { on } \Gamma_{2}, \\
\mathbf{u} & =\mathbf{g}_{D}, & & \text { on } \Gamma_{3},
\end{aligned}\right.
\end{aligned}
$$



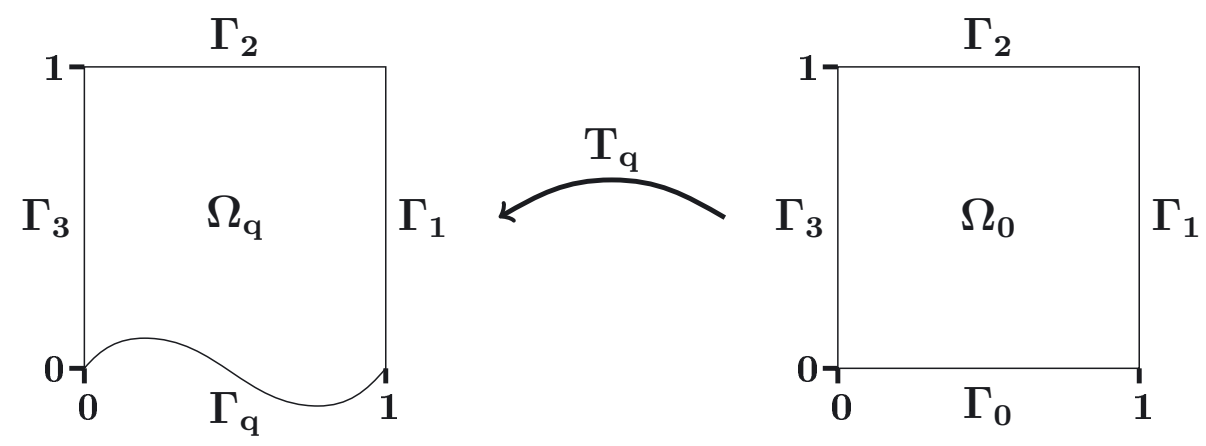

Figure 1. Physical (left) and reference (right) domains.

where $J$ is a given cost functional to be optimized, $\mathbf{u}=\left(u_{x}, u_{y}\right)$ and $p$ are the so-called state variables and $q$ is the control function (belonging to the admissible set $Q^{a d}$ ) that identifies the domain $\Omega_{q}$.

In particular, the control function $q: I=(0,1) \rightarrow \mathbb{R}$ describes the lower part $\Gamma_{q}$ of the boundary of domain $\Omega_{q}=\left\{(x, y) \in \mathbb{R}^{2} \mid x \in I, y \in(q(x), 1)\right\}$. As shown in Figure 1 (left), the boundary of $\Omega_{q}$ is partitioned as $\partial \Omega_{q}=\Gamma_{q} \cup \Gamma_{1} \cup \Gamma_{2} \cup \Gamma_{3}$.

In order to avoid domain degeneration, we fix $\varepsilon \in(0,1)$ a priori, and we introduce the following intermediate set of admissible controls ${ }^{2}$

$$
\bar{Q}^{a d}=\left\{q \in H^{3}(I) \cap H_{0}^{1}(I): q(x) \leq 1-\varepsilon, \forall x \in I\right\} .
$$

In the following, it will be useful to have the admissible controls in a bounded set, so we fix a constant $C>0$ and reduce $\bar{Q}^{a d}$ to the following set:

$$
Q^{a d}=\left\{q \in \bar{Q}^{a d}:\|q\|_{H^{3}(I)} \leq C\right\}
$$

From the above definition, it follows that all the feasible domains $\Omega_{q}$ are contained in a bounded, convex, hold-all domain $\widehat{\Omega} \subset \mathbb{R}^{2}$.

The weak formulation of problem (2.1) reads:

$$
\text { Find } \mathbf{u}=\widetilde{\mathbf{u}}+\widetilde{\mathcal{R}} \mathbf{g}_{D}, \widetilde{\mathbf{u}} \in V_{q} \text { and } \widetilde{p} \in P_{q} \text { such that }
$$

$$
\left\{\begin{aligned}
a_{q}(\widetilde{\mathbf{u}}, \mathbf{v})+b_{q}(\mathbf{v}, \widetilde{p}) & =F_{q}(\mathbf{v}), & & \forall \mathbf{v} \in V_{q}, \\
b_{q}(\widetilde{\mathbf{u}}, \pi) & =-b_{q}\left(\widetilde{\mathcal{R}} \mathbf{g}_{D}, \pi\right), & & \forall \pi \in P_{q},
\end{aligned}\right.
$$

where

$$
\begin{aligned}
& V_{q}=\left\{\mathbf{v} \in\left[H^{1}\left(\Omega_{q}\right)\right]^{2}: \mathbf{v}=\left(v_{x}, v_{y}\right)=\mathbf{0} \text { on } \Gamma_{3} \cup \Gamma_{q} \text { and } v_{y}=0 \text { on } \Gamma_{2}\right\}, \\
& P_{q}=L^{2}\left(\Omega_{q}\right),
\end{aligned}
$$

\footnotetext{
${ }^{2}$ Shape optimization for more general classes of admissible domains, and governed by more general differential problems, has been studied in the literature (see, e.g., $[20,33]$ ). Anyway, the restrictions we make on the set of admissible controls are functional to obtain estimates and convergence rates on the discretization errors of the quantities involved in our shape optimization problem (see Sect. 3).
} 
and

$$
\begin{aligned}
a_{q}(\mathbf{u}, \mathbf{v}) & =\int_{\Omega_{q}}(\eta \mathbf{u} \cdot \mathbf{v}+\nu \nabla \mathbf{u} \cdot \nabla \mathbf{v}) \mathrm{d} \Omega, \\
b_{q}(\mathbf{v}, \pi) & =-\int_{\Omega_{q}} \pi \operatorname{div} \mathbf{v} \mathrm{d} \Omega, \\
F_{q}(\mathbf{v}) & =\int_{\Omega_{q}} \mathbf{f} \cdot \mathbf{v} \mathrm{d} \Omega-a_{q}\left(\widetilde{\mathcal{R}} \mathbf{g}_{D}, \mathbf{v}\right)+\left\langle\mathbf{g}_{N}, \mathbf{v}\right\rangle_{\Gamma_{1}} .
\end{aligned}
$$

Data functions $\eta, \nu$, f are defined on the hold-all domain $\widehat{\Omega},{ }^{3}$ boundary data $\mathbf{g}_{N}, \mathbf{g}_{D}$ are defined on the fixed edges $\Gamma_{1}, \Gamma_{3}$, respectively, and $\widetilde{\mathcal{R}} \mathbf{g}_{D}$ is a continuous lifting of $\mathbf{g}_{D}$ on $\Omega_{q}$.

Remark 2.1 (Well-posedness of the state problem). Using classical results on Stokes problem (see, e.g., [18]), we can ensure the well-posedness of (2.4). Concerning data functions, we assume what follows: ${ }^{4}$

- $\eta(\mathbf{x}) \geq 0, \nu(\mathbf{x}) \geq \nu_{0}>0 \quad \forall \mathbf{x} \in \widehat{\Omega}$

- $\nu, \eta \in L^{\infty}(\widehat{\Omega})$;

- $\mathbf{f} \in\left[L^{2}(\widehat{\Omega})\right]^{2}, \mathbf{g}_{D} \in\left[H^{1 / 2}\left(\Gamma_{3}\right)\right]^{2}, \mathbf{g}_{N} \in\left[H^{-1 / 2}\left(\Gamma_{1}\right)\right]^{2}$.

Under these conditions, the following stability estimate holds:

$$
\|\widetilde{\mathbf{u}}\|_{V_{q}}+\|\widetilde{p}\|_{P_{q}} \leq c\left(\|\mathbf{f}\|_{\left[L^{2}(\widehat{\Omega})\right]^{2}}+\left\|\mathbf{g}_{D}\right\|_{\left[H^{1 / 2}\left(\Gamma_{3}\right)\right]^{2}}+\left\|\mathbf{g}_{N}\right\|_{\left[H^{-1 / 2}\left(\Gamma_{1}\right)\right]^{2}}\right) .
$$

We remark that constant $c$ in (2.6) is independent of $q$, since the inf-sup constant of the form $b_{q}$ is lowerbounded, for any $q$, by the inf-sup constant related to the hold-all domain $\widehat{\Omega}$. Hence, the right-hand side of (2.6) represents an upper bound for $\|\widetilde{\mathbf{u}}\|_{V_{q}},\|\widetilde{p}\|_{P_{q}}$, uniformly on $q$.

Finally, we introduce the cost functional

$$
J(q, \widetilde{\mathbf{u}}, \widetilde{p})=\int_{\Omega_{q}}|\nabla \widetilde{\mathbf{u}}|^{2} \mathrm{~d} \Omega+\frac{\alpha}{2}\left\|q^{\prime \prime}\right\|_{L^{2}(I)}^{2}+\frac{\beta}{2}\left(\int_{I} q(x) \mathrm{d} x-\bar{V}\right)^{2},
$$

representing the total energy dissipation of the Stokes flow, with a regularization term $\left\|q^{\prime \prime}\right\|_{L^{2}(I)}^{2}$ (as in [26]) and a volume penalty term, measuring the difference between the area under the graph of $q$ and a fixed value $\bar{V} .^{5}$

Let us introduce the state solution operator $\widetilde{S}(q)$, mapping each $q \in Q^{a d}$ to the corresponding solution $\widetilde{S}(q)=(\widetilde{\mathbf{u}}, \widetilde{p})$ of $(2.4)$, together with the reduced cost functional $\widetilde{j}$ defined as follows:

$$
\widetilde{j}: Q^{a d} \rightarrow \mathbb{R}, \quad \widetilde{j}(q):=J(q, \widetilde{S}(q)) .
$$

For convenience, it can be useful to define the following constants, whose existence is ensured by the fact that $q$ belongs to $Q^{a d}$ :

$$
d_{1}, d_{2}>0 \text { such that }\left\|q^{\prime \prime}\right\|_{L^{\infty}(I)} \leq d_{1},\left|q^{\prime}(0)\right| \leq d_{2} .
$$

Finally, we introduce the set of admissible control variations, namely:

$$
\delta Q=\left\{\delta q \in H^{3}(I) \cap H_{0}^{1}(I): q+\delta q \in Q^{a d}, \forall q \in Q^{a d}\right\} .
$$

Remark 2.2. We point out that $Q^{a d}$ is convex, closed and bounded in $H^{3}(I)$ : boundedness is stated in $(2.3)$, whereas closure and convexity are consequences of the fact that definitions $(2.2)$ and (2.3) involve only constraints of the form $\zeta(q) \leq c$, where $c$ is a constant and $\zeta$ is either the identity or the $H^{3}$ norm: the continuity of $\zeta$ provides closure, and the triangle inequality convexity.

\footnotetext{
${ }^{3}$ If not necessary, no special notation will be used to point out whether the functions in $\widehat{\Omega}$ are to be considered, or their restrictions to $\Omega_{q}$ : the distinction will be inferable from the context.

${ }^{4}$ If a particular $q$ is fixed, the conditions need only to be respected on $\Omega_{q}$. However, in order to be free from dependence on the control, we formulate them on the hold-all domain $\widehat{\Omega}$.

${ }^{5}$ Volume constraints are typical of shape optimization for fluid dynamics: see, e.g., [30,32].
} 


\subsection{Domain transformation}

In this section, we map the original problem (2.1) onto a reference domain. The main advantage of this technique lays in the numerical solution of the optimization problem: solving the state problem on a reference domain avoids the need to deform the computational mesh at each step of the optimization algorithm.

Let us introduce the reference domain $\Omega_{0}=(0,1)^{2}$, which is equivalent to the choice $q \equiv 0$. It follows that any admissible domain $\Omega_{q}$ can be seen as a transformation of $\Omega_{0}$ by means of the map

$$
T_{q}: \Omega_{0} \rightarrow \Omega_{q}, \quad \text { with } T_{q}(x, y)=\left(\mathbb{1}+\mathscr{T}_{q}\right)(x, y)=\left(\begin{array}{c}
x \\
y+(1-y) q(x)
\end{array}\right),
$$

where $\mathscr{T}_{q}$ denotes the displacement field $(0,(1-y) q(x))^{T}$. We denote by $(\cdot, \cdot)$ the $L^{2}$ inner product on $\Omega_{0}$, while $(\cdot, \cdot)_{I}$ and $(\cdot, \cdot)_{\Omega_{q}}$ indicate the scalar product in $L^{2}(I)$ and $L^{2}\left(\Omega_{q}\right)$, respectively.

Remark 2.3 (Notation I). We will use the following quantities depending on $T_{q}$ :

Map gradient: $D T_{q} \quad$ with $\quad\left(D T_{q}\right)_{i j}=\partial_{x_{j}}\left(T_{q}\right)_{i}, \quad i, j=1,2$.

Map jacobian: $\gamma_{q}=\operatorname{det}\left(D T_{q}\right)$.

Laplacian-related matrix: $A_{q}=\gamma_{q} D T_{q}^{-1} D T_{q}^{-T}$.

Remark 2.4 (Notation II). By the superscript. ${ }^{q}$ we denote the composition with the map $T_{q}$. On the other hand, whenever no doubt arises on which $q$ is considered, the composition with the inverse map $T_{q}^{-1}$ will be denoted by $\stackrel{\text { ? }}{0}$

We are now ready to state the variational problem (2.4) on the pulled-back spaces $V$ and $P$, that do not depend anymore on $q$ :

Find $(\mathbf{u}, p) \in V \times P$, such that

$$
\left\{\begin{aligned}
a(q)(\mathbf{u}, \mathbf{v})+b(q)(\mathbf{v}, p) & =F(q)(\mathbf{v}), & & \forall \mathbf{v} \in V, \\
b(q)(\mathbf{u}, \pi) & =G(q)(\pi), & & \forall \pi \in P,
\end{aligned}\right.
$$

where

$$
\begin{aligned}
& V=\left\{\mathbf{v} \in\left[H^{1}\left(\Omega_{0}\right)\right]^{2}: \mathbf{v}=\left(v_{x}, v_{y}\right)=\mathbf{0} \text { on } \Gamma_{3} \cup \Gamma_{0} \text { and } v_{y}=0 \text { on } \Gamma_{2}\right\}, \\
& P=L^{2}\left(\Omega_{0}\right),
\end{aligned}
$$

and

$$
\begin{aligned}
a(q)(\mathbf{u}, \mathbf{v}) & =\int_{\Omega_{0}}\left[\eta^{q} \mathbf{u} \cdot \mathbf{v} \gamma_{q}+\nu^{q} \operatorname{tr}\left(\nabla \mathbf{u} A_{q} \nabla \mathbf{v}^{T}\right)\right] \mathrm{d} \Omega \\
b(q)(\mathbf{v}, \pi) & =-\int_{\Omega_{0}} \pi \operatorname{tr}\left(\nabla \mathbf{v} D T_{q}^{-1}\right) \gamma_{q} \mathrm{~d} \Omega \\
F(q)(\mathbf{v}) & =\int_{\Omega_{0}} \mathbf{f}^{q} \cdot \mathbf{v} \gamma_{q} \mathrm{~d} \Omega-a(q)\left(\mathcal{R} \mathbf{g}_{D}, \mathbf{v}\right)+\left\langle\mathbf{g}_{N}, \mathbf{v}\right\rangle_{\Gamma_{1}}, \\
G(q)(\pi) & =-b(q)\left(\mathcal{R}_{D}, \mathbf{v}\right) .
\end{aligned}
$$

Remark 2.5 (Lifting). $\mathcal{R} \mathbf{g}_{D}$ represents a continuous lifting of the Dirichlet datum $\mathbf{g}_{D}$ onto $\Omega_{0}$. However, as $\mathbf{g}_{D}$ is defined on $\Gamma_{3}$, where $T_{q}$ is equal to the identity, it does not need to be mapped onto the reference domain. The fact that, in general, $\mathcal{R} \mathbf{g}_{D} \neq \widetilde{\mathcal{R}} \mathbf{g}_{D} \circ T_{q}$, does not represent an obstruction, since in the following we are not making use of any explicit expression of the lifting. 
Finally, we introduce the solution operator $S: Q^{a d} \rightarrow V \times P$, which maps an admissible control function to the solution of the transformed state problem (2.8). It follows that the original optimization problem can be reformulated as follows:

Find $\bar{q} \in Q^{a d}$ such that

$$
j(\bar{q})=\min _{q \in Q^{a d}} j(q)=\min _{q \in Q^{a d}} J\left(q, S(q) \circ T_{q}^{-1}\right) .
$$

This is the formulation we will refer to on the rest of the paper.

\subsection{Well-posedness of the problem}

In this section, we analyze the well-posedness of the state problem (2.8) and the existence of an optimal solution to our minimization problem (2.9). We remark that, since (2.8) is obtained by applying to (2.4) the change of variables induced by the map $T_{q}$, the solution $(\mathbf{u}, p)$ to problem $(2.8)$ and the solution $(\widetilde{\mathbf{u}}, \widetilde{p})$ to problem (2.4) are connected by the following identities:

$$
\mathbf{u}=\widetilde{\mathbf{u}} \circ T_{q}, \quad p=\widetilde{p} \circ T_{q} .
$$

Therefore, it is not restrictive to focus the analysis on the transformed problem (2.8). Indeed, the well-posedness of the original problem (2.4) is equivalent to that of (2.8), due to the equivalence in $L^{2}\left(\Omega_{q}\right)$ of the norms $\varphi \mapsto\|\varphi\|_{L^{2}\left(\Omega_{q}\right)}$ and $\varphi \mapsto\left\|\varphi \circ T_{q}\right\|_{L^{2}\left(\Omega_{0}\right)}$.

At first, we observe that matrix $A_{q}$ belongs to $\left[L^{\infty}\left(\Omega_{0}\right)\right]^{2 \times 2}$, it is symmetric and positive definite, and its eigenvalues are lower-bounded by

$$
\lambda_{0}=2\left(1+\frac{1+\left(d_{1}+d_{2}\right)^{2}}{\varepsilon}+{\sqrt{\left(1+\frac{1+\left(d_{1}+d_{2}\right)^{2}}{\varepsilon}\right)^{2}-4}}^{-1}>0 .\right.
$$

Under the same assumptions of Remark 2.1, the coercivity of the form $a(q)$ and the continuity of the functionals and forms involved in (2.8) are given by the following inequalities, holding for any $\mathbf{u}, \mathbf{v} \in V, \pi \in P, q \in Q^{a d}$ :

$$
\begin{aligned}
a(q)(\mathbf{v}, \mathbf{v}) \geq & \nu_{0} \lambda_{0}\|\nabla \mathbf{v}\|^{2}=: \alpha_{c}\|\nabla \mathbf{v}\|^{2} \\
|a(q)(\mathbf{u}, \mathbf{v})| \leq & \left(\|\eta\|_{L^{\infty}(\widehat{\Omega})}\left\|\gamma_{q}\right\|_{\infty}+\|\nu\|_{L^{\infty}(\widehat{\Omega})} \frac{1}{\lambda_{0}}\right)\|\nabla \mathbf{u}\|\|\nabla \mathbf{v}\| \\
\leq & \left(\|\eta\|_{L^{\infty}(\widehat{\Omega})}\left(1+d_{1}+d_{2}\right)+\|\nu\|_{L^{\infty}(\widehat{\Omega})} \frac{1}{\lambda_{0}}\right)\|\nabla \mathbf{u}\|\|\nabla \mathbf{v}\|=: M\|\nabla \mathbf{u}\|\|\nabla \mathbf{v}\|, \\
|b(q)(\mathbf{v}, \pi)| \leq & \left\|\gamma_{q} D T_{q}^{-T}\right\|_{\infty}\|\nabla \mathbf{v}\|\|\pi\| \leq 2\left(1+d_{1}+d_{2}\right)\|\nabla \mathbf{v}\|\|\pi\|=: M_{b}\|\nabla \mathbf{v}\|\|\pi\|, \\
|F(q)(\mathbf{v})| \leq & \left\|\gamma_{q}\right\|_{\infty}\|\mathbf{f}\|_{\left[L^{2}(\widehat{\Omega})\right]^{2}}\|\mathbf{v}\|+M c_{\mathcal{R}}\left\|\mathbf{g}_{D}\right\|_{\left[H^{1 / 2}\left(\Gamma_{3}\right)\right]^{2}}\|\nabla \mathbf{v}\| \\
& +\left\|\mathbf{g}_{N}\right\|_{\left[H^{-1 / 2}\left(\Gamma_{1}\right)\right]^{2}} c_{t r}\|\nabla \mathbf{v}\| \\
\leq & {\left[c_{\widehat{\Omega}}\left(1+d_{1}+d_{2}\right)\|\mathbf{f}\|_{\left[L^{2}(\widehat{\Omega})\right]^{2}}+M c_{\mathcal{R}}\left\|\mathbf{g}_{D}\right\|_{\left[H^{1 / 2}\left(\Gamma_{3}\right)\right]^{2}}\right.} \\
& \left.+\left\|\mathbf{g}_{N}\right\|_{\left[H^{-1 / 2}\left(\Gamma_{1}\right)\right]^{2}} c_{t r}\right]\|\nabla \mathbf{v}\| \\
= & : M_{F}\|\nabla \mathbf{v}\|,
\end{aligned}
$$

where the constants $\alpha_{c}, M, M_{b}, M_{F}$ are independent of $q$.

The inf-sup condition for problem (2.8) reads

There exists a positive constant $\bar{\beta}$, independent of $q$, such that

$$
\forall \pi \in P \quad \exists \mathbf{v} \in V \quad: \quad b(q)(\mathbf{v}, \pi) \geq \bar{\beta}\|\nabla \mathbf{v}\|\|\pi\| .
$$


The validity of this property would allow to exploit the classical saddle-point-problem theory also for the transformed problem (2.8).

To prove (2.11), we start considering the inf-sup condition on $\Omega_{0}$, with constant $\widehat{\beta}>0$, namely:

$$
\forall \pi \in P \quad \exists \mathbf{v} \in V \text { such that } b(0)(\mathbf{v}, \pi)=-\int_{\Omega_{0}} \pi \operatorname{div} \mathbf{v} \mathrm{d} \Omega \geq \widehat{\beta}\|\pi\|\|\nabla \mathbf{v}\| .
$$

Employing the definition of $b(q)$, the following holds for any $q \in Q^{a d}$

$$
\begin{aligned}
b(q)(\mathbf{v}, \pi) & =-\int_{\Omega_{0}} \pi \nabla \mathbf{v} \cdot \gamma_{q} D T_{q}^{-T} \mathrm{~d} \Omega=-\int_{\Omega_{0}} \pi \nabla \mathbf{v} \cdot\left(\mathbb{1}+\gamma_{q} D T_{q}^{-T}-\mathbb{1}\right) \mathrm{d} \Omega \\
& \geq-\int_{\Omega_{0}} \pi \operatorname{div} \mathbf{v} \mathrm{d} \Omega-\left|\int_{\Omega_{0}} \pi \nabla \mathbf{v} \cdot\left(\gamma_{q} D T_{q}^{-T}-\mathbb{1}\right) \mathrm{d} \Omega\right| \geq\left(\widehat{\beta}-\left\|\gamma_{q} D T_{q}^{-T}-\mathbb{1}\right\|_{\infty}\right)\|\pi\|\|\nabla \mathbf{v}\|,
\end{aligned}
$$

being $\pi \in P$ and $\mathbf{v} \in V$ related through (2.12). As it holds

$$
\gamma_{q} D T_{q}^{-T}-\mathbb{1}=\operatorname{cof}\left(D T_{q}\right)-\mathbb{1}=\left(\begin{array}{cc}
-q-(1-y) q^{\prime} \\
0 & 0
\end{array}\right)
$$

we get

$$
b(q)(\mathbf{v}, \pi) \geq\left(\widehat{\beta}-\|q\|_{W^{1, \infty}(I)}\right)\|\pi\|\|\nabla \mathbf{v}\| .
$$

Therefore, requiring $\|q\|_{W^{1, \infty}(I)}$ to be strictly smaller than $\widehat{\beta}$ yields the validity of the inf-sup (2.11), uniformly on $q$.

It is easy to check (see, e.g., [14]) that on the domain $\Omega_{0}$ the inf-sup constant $\widehat{\beta}$ in (2.12) satisfies $\widehat{\beta} \geq \frac{1}{4 \sqrt{2}}$. Hence, in order to ensure the validity of $(2.11)$ it is sufficient to require

$$
\|q\|_{H^{3}(I)} \leq \frac{\xi}{8}, \text { for some } \xi \in(0,1)
$$

in the definition of the set $Q^{a d}$ of admissible controls.

Remark 2.6. We remark that condition (2.13) is representative of a class of sufficient conditions ensuring the validity of (2.11). Most likely, less stringent conditions can be found. However, real world shape optimization problems often deal with very smooth configurations, thus compatible with (2.13).

Having ensured that the bilinear form $a$ is coercive and continuous (see (2.10a) and (2.10b)) and that $b$ is continuous (see (2.10c)) and satisfies the inf-sup condition (2.11), we can finally employ the classical results of saddle-point theory to prove the following result (see, e.g., [8]):

Proposition 2.7. Under condition (2.13), for each $q \in Q^{a d}$ the pulled-back problem (2.8) admits a unique solution, and the following inequality holds

$$
\|S(q)\|_{V \times P} \leq c\left(\mathbf{f}, \mathbf{g}_{D}, \mathbf{g}_{N}, \eta, \nu, \widehat{\Omega}\right),
$$

where the constant $c$ is independent of $q$.

Concluding this section, we prove the existence of an optimal solution to (2.9). Existence results have already been proved in more general settings (see, e.g., [33], where compressible Navier-Stokes equations are taken into account), employing sophisticated analytical techniques. Anyway, for the following result we present a simpler proof, based on the direct method of calculus of variations, and holding under the assumptions defining the framework of the present paper. 
Theorem 2.8. Let $Q^{a d}$ be a non-empty, convex, closed and bounded subset of $H^{3}(I)$ and let $S: Q^{\text {ad }} \rightarrow$ $\left[H^{1}\left(\Omega_{0}\right)\right]^{2} \times L^{2}\left(\Omega_{0}\right)$ be the solution operator of problem (2.8). Then, there exists a solution to the minimization problem (2.9).

Proof. The proof follows standard ideas of calculus of variations. Hence, in the following we sketch the main steps of the proof. From Remark 2.2, we know that $Q^{a d}$ is a closed, bounded and convex subset of $H^{3}(I)$. This set is also non-empty, since $q \equiv 0$ fulfills all its constraints.

Observing that $j(q) \geq 0$ for any $q \in Q^{a d}$ and that $Q^{a d} \neq \emptyset$, we have that a minimizing sequence $\left\{q_{n}\right\}_{n \in \mathbb{N}} \subset Q^{a d}$ exists, such that

$$
\lim _{n \rightarrow \infty} j\left(q_{n}\right)=\inf _{q \in Q^{a d}} j(q)=: \bar{j} .
$$

Being $Q^{a d}$ bounded in $H^{3}(I)$, the sequence $\left\{q_{n}\right\}$ is bounded itself, then there exists a subsequence $\left\{q_{n_{k}}\right\}$ and some $\bar{q} \in H^{3}(I)$ such that,

$$
q_{n_{k}} \rightarrow \bar{q} \quad \text { in } H^{3}(I) \quad \text { for } k \rightarrow \infty .
$$

Moreover, being $Q^{a d}$ closed and convex, the limit $\bar{q}$ belongs to $Q^{a d}$.

The next step is to show that we can take the limit also in the state variables sequence $\left\{S\left(q_{n_{k}}\right)\right\}=\left\{\left(\mathbf{u}_{k}, p_{k}\right)\right\}$. For this purpose, following some ideas of the proof of ([22], Lem. 2.20), we consider the physical counterpart $\left\{\widetilde{S}\left(q_{n_{k}}\right)\right\}=\left\{S\left(q_{n_{k}}\right) \circ T_{q_{n_{k}}}^{-1}\right\}$ of the sequence and the trivial extension to zero of its elements in $\widehat{\Omega} \supset \Omega_{q}$, denoted by $\left\{\widehat{S}\left(q_{n_{k}}\right)\right\}$.

Thanks to the well-posedness of problem (2.4), uniformly on $q \in Q^{a d}$, the sequence $\left\{\widehat{S}\left(q_{n_{k}}\right)\right\}$ is bounded in $\widehat{V} \times \widehat{P}=H^{1}(\widehat{\Omega}) \times L^{2}(\widehat{\Omega})$. Hence, there exists a subsequence, for simplicity denoted by $\left\{\widehat{S}\left(q_{l}\right)\right\}$, and some $\overline{\widehat{S}}=(\overline{\widehat{\mathbf{u}}}, \overline{\widehat{p}}) \in \widehat{V} \times \widehat{P}$ such that,

$$
\widehat{S}\left(q_{l}\right)=\left(\widehat{\mathbf{u}}_{l}, \widehat{p}_{l}\right) \rightarrow \overline{\widehat{S}}=(\overline{\widehat{\mathbf{u}}}, \overline{\widehat{p}}) \text { in } \widehat{V} \times \widehat{P} \quad \text { for } l \rightarrow \infty .
$$

Now we have to prove that $\bar{S}:=\left.\overline{\widehat{S}}\right|_{\Omega_{\bar{q}}} \circ T_{\bar{q}}$ is the state solution corresponding to $\bar{q}$, i.e. $\bar{S}=S(\bar{q})$. This can be done transforming each term in problem (2.8) back on $\Omega_{q_{l}}$, extending it on $\widehat{\Omega}$ and then passing to the limit for $l \rightarrow \infty$. As a paradigmatic example, we consider the viscosity term. Let us take $\mathbf{v} \in V_{\bar{q}}$ (see (2.5) for the definition of the space) and its trivial extension $\widehat{\mathbf{v}}$ to $\widehat{\Omega}$, and let us denote by $\chi_{l}, \bar{\chi}$ the characteristic functions of the domains $\Omega_{q_{l}}, \Omega_{\bar{q}}$, respectively. We want to prove that the following tends to zero, for $l \rightarrow \infty$ :

$$
\begin{gathered}
\left|\int_{\Omega_{q_{l}}} \nu \nabla \widetilde{\mathbf{u}}_{l} \cdot \nabla \widehat{\mathbf{v}} \mathrm{d} \Omega-\int_{\Omega_{\bar{q}}} \nu \nabla \overline{\widetilde{\mathbf{u}}} \cdot \nabla \widehat{\mathbf{v}} \mathrm{d} \Omega\right|=\left|\int_{\widehat{\Omega}} \chi_{l} \nu \nabla \widehat{\mathbf{u}}_{l} \cdot \nabla \widehat{\mathbf{v}} \mathrm{d} \Omega-\int_{\widehat{\Omega}} \bar{\chi} \nu \nabla \overline{\mathbf{u}} \cdot \nabla \widehat{\mathbf{v}} \mathrm{d} \Omega\right| \\
\leq\left|\int_{\widehat{\Omega}} \chi_{l} \nu \nabla \widehat{\mathbf{v}} \cdot\left(\nabla \widehat{\mathbf{u}}_{l}-\nabla \overline{\mathbf{u}}\right) \mathrm{d} \Omega\right|+\left|\int_{\widehat{\Omega}}\left(\chi_{l}-\bar{\chi}\right) \nu \nabla \widehat{\mathbf{v}} \cdot \nabla \overline{\mathbf{u}} \mathrm{d} \Omega\right| .
\end{gathered}
$$

To prove the nullity of the limit of (2.14), we remind that $q_{l} \rightarrow \bar{q}$ in $H^{3}(I)$, then, in particular, we have that $q_{l} \rightarrow \bar{q}$ point-wise, and as a consequence $\chi_{l}(\mathbf{x}) \rightarrow \bar{\chi}(\mathbf{x}) \forall \mathbf{x} \in \widehat{\Omega}$. Being $\nu \in L^{\infty}(\widehat{\Omega})$, it holds that the whole integrand of the last term in (2.14) belongs to $L^{1}(\widehat{\Omega})$, and then we can employ the dominated convergence theorem to prove that the integral tends to zero. For the first term in the right-hand side of (2.14), we use the fact that

$$
\text { since } \nabla \widehat{\mathbf{u}}_{l}-\nabla \overline{\mathbf{u}} \rightarrow 0, \chi_{l} \nu \nabla \widehat{\mathbf{v}} \rightarrow \bar{\chi} \nu \nabla \widehat{\mathbf{v}} \text { in }\left[L^{2}(\widehat{\Omega})\right]^{2 \times 2}, \quad \text { then }\left(\nabla \widehat{\mathbf{u}}_{l}-\nabla \overline{\mathbf{u}}, \chi_{l} \nu \nabla \widehat{\mathbf{v}}\right)_{\widehat{\Omega}} \rightarrow(0, \bar{\chi} \nu \nabla \widehat{\mathbf{v}})_{\widehat{\Omega}},
$$

where the strong convergence of $\chi_{l} \nu \nabla \widehat{\mathbf{v}}$ is due to the point-wise convergence of $\chi_{l}$ to $\chi$ and the dominated convergence theorem in $\left[L^{2}(\widehat{\Omega})\right]^{2 \times 2}$.

Since for any $l \in \mathbb{N}$ the restriction $\left.\widehat{\mathbf{v}}\right|_{\Omega_{q_{l}}}$ can be approximated by functions belonging to $V_{q_{l}}$ (see, e.g., the proof of ([22], Lem. 2.20)), the limit taken in (2.14), together with the ones involving the other terms of problem (2.4), 
allows to take the limit in the whole problem (2.4) with $q=q_{l}$, yielding that $\overline{\widetilde{S}}=\left.\overline{\widehat{S}}\right|_{\Omega_{\bar{q}}}$ actually is the solution to the limit problem. Therefore, its composition with the limit domain map, $\bar{S}=\overline{\widetilde{S}} \circ T_{\bar{q}}$, is the solution to the pulled-back problem (2.8) with $q=\bar{q}$.

Finally, using once again the dominated convergence theorem and the weak, lower semi-continuity of seminorms in a Banach space yields the weak, lower semi-continuity of functional $j$, allowing to conclude that

$$
j\left(q_{l}\right) \rightarrow j(\bar{q})=\bar{j} \quad \text { for } l \rightarrow \infty .
$$

Hence $\bar{q}$ turns out to be a solution to the optimization problem (2.9).

Remark 2.9. In general, we cannot expect the optimal control $\bar{q}$ to be unique. Indeed, even if the state problem (2.8) is linear, the solution operator $S$ is nonlinear, and the functional $j$ is in general non-convex. As a consequence, at the level of numerical approximation this lack of uniqueness requires the use of local minimization algorithms.

\section{A PRIORI ERROR ESTIMATES}

In this section, we aim at deriving some a priori estimates for the numerical discretization error of the main quantities involved in our problem, namely the control function $q$, the state variable $S(q)$ and the reduced cost functional $j(q)$.

At first, we are going to discuss some differentiability properties of the state solution operator $S$, under suitable assumptions (Cor. 3.2, Thm. 3.5). Then, we will introduce a discretization on the control space and derive corresponding error estimates (Prop. 3.6). Afterwards, the discretization of the state problem will be studied (Lems. 3.19 and 3.20). Finally, we will derive a convergence result for the complete shape optimization problem (Thm. 3.21).

\subsection{Solution operator properties}

In order to provide some differentiability properties for the state solution operator and the cost functional, we begin by considering the following generalization of the Implicit Function Theorem to Banach spaces:

Theorem 3.1 ([26], Thm. 3.3). Let $\mathcal{F} \in C^{k}\left(X^{a d} \times Y, Z\right), k \geq 1$, where $Y$ and $Z$ are Banach spaces and $X^{\text {ad }}$ is an open subset of Banach space $X$. Suppose that $\mathcal{F}\left(x^{*}, y^{*}\right)=0$ and $\mathcal{F}_{y}^{\prime}\left(x^{*}, y^{*}\right)$ is continuously invertible. Then there exist neighborhoods $\Theta$ of $x^{*}$ in $X$, $\Phi$ of $y^{*}$ in $Y$ and a map $g \in C^{k}(\Theta, Y)$ such that $\mathcal{F}(x, g(y))=0$ for all $x \in \Theta$. Furthermore, $F(x, y)=0$ for $(x, y) \in \Theta \times \Phi$ implies $y=g(x)$.

As a direct consequence, we can prove the following result:

Corollary 3.2. Let the following assumptions hold:

$$
\eta, \nu \in C^{2}(\overline{\widehat{\Omega}}), \quad \mathbf{f} \in\left[C^{2}(\overline{\widehat{\Omega}})\right]^{2} .
$$

Then, the solution operator $S$ is at least twice continuously Fréchet-differentiable.

Proof. It is enough to use Theorem 3.1, with $X=H^{3}(I) \cap H_{0}^{1}(I), Y=V \times P, Z=Y^{\prime}$, the open set $X^{a d}=$ $\operatorname{int}\left(Q^{a d}\right)$ and the map $\mathcal{F}: X^{a d} \times Y \rightarrow Z$ such that

$$
\mathcal{F}(q ; \mathbf{u}, p)=\left(\begin{array}{c}
a(q)(\mathbf{u}, \cdot)+b(q)(\cdot, p)-F(q)(\cdot) \\
b(q)(\mathbf{u}, \cdot)-G(q)(\cdot)
\end{array}\right) \text { for any } q \in \operatorname{int}\left(Q^{a d}\right), \mathbf{u} \in V, p \in P .
$$

Being each component of the map $\mathcal{F}$ a linear combination of the forms $a, b, F, G$, we deduce that $\mathcal{F} \in C^{2}\left(X^{a d} \times\right.$ $Y, Z)$ if we prove that each form is $C^{2}$. Let us detail the proof only for the application $(q, \mathbf{u}) \mapsto a(q)(\mathbf{u}, \cdot)$, since the regularity of the other forms can be proved by similar arguments. 
Being the form $a(q)(\cdot, \cdot)$ bilinear for any value of $q \in Q^{a d}$, and bounded if seen as a functional of its first argument, the dependence of the form on $\mathbf{u}$ is $C^{\infty}$. Regarding the dependence on $q$, instead, we have to consider the regularity of the coefficients. In particular, we have that the applications $q \mapsto T_{q}$ and $q \mapsto A_{q}$ belong to $C^{\infty}\left(Q^{a d}\right)$, as one can infer from the definition of $T_{q}$ and $A_{q}$, and hence, under the hypothesis (3.1), we deduce that the applications $q \mapsto \eta^{q}$ and $q \mapsto \nu^{q}$ are $C^{2}$. Therefore, $a(q)(\mathbf{u}, \cdot)$ is $C^{2}$ also as a function of $q$.

Now, we show that $\mathcal{F}_{y}^{\prime}(x, y)$ is continuously invertible for any $(x, y) \in X^{a d} \times Y$. The computation of this derivative along the direction $(\delta \mathbf{u}, \delta p)$ provides the following identity:

$$
\mathcal{F}_{y}^{\prime}(q ; \mathbf{u}, p)(\delta \mathbf{u}, \delta p)=\left(\begin{array}{c}
a(q)(\delta \mathbf{u}, \cdot)+b(q)(\cdot, \delta p) \\
b(q)(\delta \mathbf{u}, \cdot)
\end{array}\right) \text { for any } q \in \operatorname{int}\left(Q^{a d}\right), \mathbf{u}, \delta \mathbf{u} \in V, p, \delta p \in P .
$$

It follows that the invertibility and bicontinuity of the operator $(\delta \mathbf{u}, \delta p) \mapsto \mathcal{F}_{y}^{\prime}(q ; \mathbf{u}, p)(\delta \mathbf{u}, \delta p)$ is equivalent to the well-posedness of the homogeneous version of the pulled-back Stokes problem (2.8), which has been proved in Proposition 2.7.

Comparing the definition of $\mathcal{F}$ with problem (2.8), we can see that for any $\left(q^{*} ; \mathbf{u}^{*}, p^{*}\right) \in X^{a d} \times Y$, problem (2.8) is equivalent to the equation $\mathcal{F}\left(q^{*} ; \mathbf{u}^{*}, p^{*}\right)=0$, hence this equation can be rewritten as $\mathcal{F}\left(q^{*}, S\left(q^{*}\right)\right)=0$. Therefore, the operator $S$ corresponds to the map $g$ defined in Theorem 3.1. Hence, invoking Theorem 3.1 with $k=2$, the regularity result holding for $g$ holds for $S$ as well. Moreover, since we can apply Theorem 3.1 to any element $\left(x^{*}, y^{*}\right)=\left(q^{*}, \mathbf{u}^{*}, p^{*}\right) \in X^{a d} \times Y=\operatorname{int}\left(Q^{a d}\right) \times V \times P$, the regularity of $S$ can be extended to the whole set $\operatorname{int}\left(Q^{a d}\right)$.

Remark 3.3. The proof of Corollary 3.2 holds in the particular framework of the present paper, and it has been reported for completeness. More general results on material differentiability can be found in literature (see, e.g., $[6,25,33]$ and the references therein), holding under less stringent assumptions and obtained resorting to more refined properties, such as the Hadamard differentiability of metric projections in Banach spaces.

Now, let us collect some properties of the map $T_{q}$.

Proposition 3.4. Given $q, r \in Q^{a d}$, the maps defined in Remark 2.3, depending on $T_{q}, T_{r}$ and their derivatives, satisfy the following inequalities, for any admissible variation $\delta q \in \delta Q$ :

(1) $\left\|\gamma_{q, \delta q}^{\prime}\right\|_{\infty}=\left\|\operatorname{div}\left(\mathscr{T}_{\delta q}\right)\right\|_{\infty} \leq c\|\delta q\|_{L^{\infty}(I)} \leq \bar{c}\|\delta q\|_{H^{1}(I)} ;$

(2) $\left\|\mathscr{T}_{\delta q}\right\|_{\infty} \leq c\|\delta q\|_{H^{1}(I)}$;

(3) $\left\|\operatorname{cof}\left(D \mathscr{T}_{\delta q}\right)\right\|_{\infty}=\left\|D \mathscr{T}_{\delta q}\right\|_{\infty} \leq c\|\delta q\|_{H^{2}(I)}$;

(4) $\left\|A_{q, \delta q}^{\prime}\right\|_{\infty} \leq c\|\delta q\|_{H^{2}(I)}$;

(5) $\left\|A_{q, \delta q, \delta q}^{\prime \prime}\right\|_{\infty} \leq c\|\delta q\|_{H^{2}(I)}^{2}$;

(6) $\left\|A_{q}-A_{r}\right\|_{\infty} \leq c\|q-r\|_{H^{2}(I)}$;

(7) $\left\|A_{q, \delta q}^{\prime}-A_{r, \delta q}^{\prime}\right\|_{\infty} \leq c\|q-r\|_{H^{2}(I)}\|\delta q\|_{H^{2}(I)}$;

(8) $\left\|A_{q, \delta q, \delta q}^{\prime \prime}-A_{r, \delta q, \delta q}^{\prime \prime}\right\|_{\infty} \leq c\|q-r\|_{H^{2}(I)}\|\delta q\|_{H^{2}(I)}^{2}$;

where the constants $c$ and $\bar{c}$ are independent of $q$ and $\delta q$.

Proof. Each of the present results simply follows from the bounding of the explicit expression of the quantity to be controlled, in terms of the variation $\delta q$, and from the continuous embedding of $H^{2}(I)$ in $W^{k, p}(I)$ for $k=0,1$ and $p=1,2, \infty$. The proof of bounds (1)-(3) is detailed in ([17], p. 30), inequalities (4) - (6) are stated in ([26], Lem. 1.7), while bounds (7)-(8) can be proved analyzing each entry of the matrices, separately. In the sequel, we explicitly prove (7). The expression of $A_{q, \delta q}^{\prime}$ is the following:

$$
A_{q, \delta q}^{\prime}(x, y)=\left(\begin{array}{cc}
-\delta q(x) & -(1-y) \delta q^{\prime}(x) \\
-(1-y) \delta q^{\prime}(x) & \frac{\delta q(x)+2(1-y)^{2} q^{\prime}(x) \delta q^{\prime}(x)(1-q(x))+(1-y)^{2} q^{\prime}(x)^{2} \delta q(x)}{(1-q(x))^{2}}
\end{array}\right) .
$$


Since only the entry in position $(2,2)$ depends on $q$, this is the only term giving a nonzero contribution to $\left\|A_{q, \delta q}^{\prime}-A_{r, \delta q}^{\prime}\right\|_{\infty}$. Employing the boundedness of $\Omega_{0}$ and the definition of $Q^{a d}$ in $(2.2)-(2.3)$ together with some algebraic manipulations provides us with the estimate:

$$
\begin{aligned}
&\left\|A_{q, \delta q}^{\prime}-A_{r, \delta q}^{\prime}\right\|_{\infty}=\left\|\left(A_{q, \delta q}^{\prime}\right)_{2,2}-\left(A_{r, \delta q}^{\prime}\right)_{2,2}\right\|_{\infty} \\
& \leq c_{1}\|\delta q\|_{L^{\infty}(I)}\left(\left\|(1-r)^{2}-(1-q)^{2}\right\|_{L^{\infty}(I)}+\left\|q^{\prime 2}-r^{\prime 2}\right\|_{L^{\infty}(I)}\right) \\
&+c_{2}\left\|\delta q^{\prime}\right\|_{L^{\infty}(I)}\left(\|(1-r)-(1-q)\|_{L^{\infty}(I)}+\left\|q^{\prime}-r^{\prime}\right\|_{L^{\infty}(I)}\right) \\
& \leq c\|\delta q\|_{W^{1, \infty}(I)}\|q-r\|_{W^{1, \infty}(I)} \leq c\|\delta q\|_{H^{2}(I)}\|q-r\|_{H^{2}(I)} .
\end{aligned}
$$

The inequality (8) can be proved in a completely analogous way.

Thanks to Corollary 3.2, we know that the solution operator $S$ is twice-differentiable; now, we want to explicitly write the variational problems defining its first and second variation w.r.t. $q$.

The first and second variations of the solution operator $S$ along the directions $\delta q, \tau q \in \delta Q$ are defined as follows:

(1) $S^{\prime}(q)(\delta q)=(\delta \mathbf{u}, \delta p) \in V \times P$, where $(\delta \mathbf{u}, \delta p)$ is the solution of

$$
\left\{\begin{aligned}
a(q)(\delta \mathbf{u}, \mathbf{v})+b(q)(\mathbf{v}, \delta p) & =\dot{F}(q, \delta q)(\mathbf{v})-\dot{a}(q, \delta q)(\mathbf{u}, \mathbf{v})-\dot{b}(q, \delta q)(\mathbf{v}, p) & & \forall \mathbf{v} \in V, \\
b(q)(\delta \mathbf{u}, \pi) & =\dot{G}(q, \delta q)(\pi)-\dot{b}(q, \delta q)(\mathbf{u}, \pi) & & \forall \pi \in P .
\end{aligned}\right.
$$

(2) $S^{\prime \prime}(q)(\delta q, \tau q)=(\tau \delta \mathbf{u}, \tau \delta p) \in V \times P$, where $(\tau \delta \mathbf{u}, \tau \delta p)$ is the solution of

$$
\begin{cases}a(q)(\tau \delta \mathbf{u}, \mathbf{v})+b(q)(\mathbf{v}, \tau \delta p)= & \\ \ddot{F}(q, \delta q, \tau q)(\mathbf{v})-\ddot{a}(q, \delta q, \tau q)(\mathbf{u}, \mathbf{v})-\ddot{b}(q, \delta q, \tau q)(\mathbf{v}, p) & \forall \mathbf{v} \in V, \\ -\dot{a}(q, \delta q)(\tau \mathbf{u}, \mathbf{v})-\dot{b}(q, \delta q)(\mathbf{v}, \tau p)-\dot{a}(q, \tau q)(\delta \mathbf{u}, \mathbf{v})-\dot{b}(q, \tau q)(\mathbf{v}, \delta p) & \\ b(q)(\tau \delta \mathbf{u}, \pi)=\ddot{G}(q, \delta q, \tau q)(\pi)-\ddot{b}(q, \delta q, \tau q)(\mathbf{u}, \pi) & \forall \pi \in P, \\ -\dot{b}(q, \delta q)(\tau \mathbf{u}, \pi)-\dot{b}(q, \tau q)(\delta \mathbf{u}, \pi) & \end{cases}
$$

with $(\tau \mathbf{u}, \tau p)=S^{\prime}(q)(\tau q)$.

The forms and functionals employed in (3.2) and (3.3) are defined as follows:

$$
\begin{aligned}
\dot{F}(q, \delta q)(\mathbf{v})= & \int_{\Omega_{0}}\left(\gamma_{q, \delta q}^{\prime} \mathbf{f}^{q} \cdot \mathbf{v}+\gamma_{q} \nabla \mathbf{f}^{q} \mathscr{T}_{\delta q} \cdot \mathbf{v}\right) \mathrm{d} \Omega-\dot{a}(q, \delta q)\left(\mathcal{R} \mathbf{g}_{D}, \mathbf{v}\right), \\
\dot{G}(q, \delta q)(\pi)= & -\dot{b}(q, \delta q)\left(\mathcal{R} \mathbf{g}_{D}, \pi\right) \\
\dot{a}(q, \delta q)(\mathbf{u}, \mathbf{v})= & \int_{\Omega_{0}}\left[\left(\gamma_{q} \nabla \eta^{q} \cdot \mathscr{T}_{\delta q}+\eta^{q} \gamma_{q, \delta q}^{\prime}\right) \mathbf{u} \cdot \mathbf{v}\right. \\
& \left.+\nabla \nu^{q} \cdot \mathscr{T}_{\delta q} \operatorname{tr}\left(\nabla \mathbf{u} A_{q} \nabla \mathbf{v}^{T}\right)+\nu^{q} \operatorname{tr}\left(\nabla \mathbf{u} A_{q, \delta q}^{\prime} \nabla \mathbf{v}^{T}\right)\right] \mathrm{d} \Omega, \\
\dot{b}(q, \delta q)(\mathbf{v}, \pi)= & -\int_{\Omega_{0}} \pi \nabla \mathbf{v} \cdot \operatorname{cof}\left(\mathscr{T}_{\delta q}\right) \mathrm{d} \Omega \\
\ddot{F}(q, \delta q, \tau q)(\mathbf{v})= & \int_{\Omega_{0}}\left[\gamma_{q, \delta q, \tau q}^{\prime \prime} \mathbf{f}^{q} \cdot \mathbf{v}+\gamma_{q, \delta q}^{\prime} \nabla \mathbf{f}^{q} \mathscr{T}_{\tau q} \cdot \mathbf{v}+\gamma_{q, \tau q}^{\prime} \nabla \mathbf{f}^{q} \mathscr{T}_{\delta q} \cdot \mathbf{v}\right. \\
& \left.+\gamma_{q}\left(\widetilde{\nabla}^{2} \mathbf{f}^{q} \mathscr{T}_{\tau q}+\nabla \mathbf{f}^{q} D \mathscr{T}_{\tau q}\right) \mathscr{T}_{\delta q} \cdot \mathbf{v}\right] \mathrm{d} \Omega-\ddot{a}(q, \delta q)\left(\mathcal{R} \mathbf{g}_{D}, \mathbf{v}\right), \\
\ddot{G}(q, \delta q, \tau q)(\pi)= & -\ddot{b}(q, \delta q, \tau q)\left(\mathcal{R} \mathbf{g}_{D}, \pi\right)=0,
\end{aligned}
$$




$$
\begin{aligned}
\ddot{a}(q, \delta q, \tau q)(\mathbf{u}, \mathbf{v})= & \int_{\Omega_{0}}\left\{\left[\gamma_{q, \tau q}^{\prime} \nabla \eta^{q} \cdot \mathscr{T}_{\delta q}+\left(\nabla^{2} \eta^{q} \mathscr{T}_{\tau q}+D \mathscr{T}_{\tau q}^{T} \nabla \eta^{q}\right) \cdot \mathscr{T}_{\delta q} \gamma_{q}\right.\right. \\
& \left.+\eta^{q} \gamma_{q, \delta q, \tau q}^{\prime \prime}+\gamma_{q, \delta q}^{\prime} \nabla \eta^{q} \cdot \mathscr{T}_{\tau q}\right] \mathbf{u} \cdot \mathbf{v} \\
& +\left(\nabla^{2} \nu^{q} \mathscr{T}_{\delta q}+D \mathscr{T}_{\tau q}^{T} \nabla \nu^{q}\right) \cdot \mathscr{T}_{\delta q} \operatorname{tr}\left(\nabla \mathbf{u} A_{q} \nabla \mathbf{v}^{T}\right) \\
& +\nabla \nu^{q} \cdot \mathscr{T}_{\delta q} \operatorname{tr}\left(\nabla \mathbf{u} A_{q, \tau q}^{\prime} \nabla \mathbf{v}^{T}\right)+\nu^{q} \operatorname{tr}\left(\nabla \mathbf{u} A_{q, \delta q, \tau q}^{\prime \prime} \nabla \mathbf{v}^{T}\right) \\
& \left.+\nabla \nu^{q} \cdot \mathscr{T}_{\tau q} \operatorname{tr}\left(\nabla \mathbf{u} A_{q, \delta q}^{\prime} \nabla \mathbf{v}^{T}\right)\right\} \mathrm{d} \Omega, \\
\ddot{b}(q, \delta q, \tau q)(\mathbf{v}, \pi)= & 0,
\end{aligned}
$$

with the differential operator $\widetilde{\nabla}^{2}$ acting as $\left(\widetilde{\nabla}^{2} \varphi\right)_{i j k}=\frac{\partial^{2} \varphi_{i}}{\partial x_{k} \partial x_{j}}$ and the over-signed dots denoting the partial Gateaux derivative w.r.t. the control $q$.

Theorem 3.5. Problems (3.2) and (3.3) are well-posed, and the following stability estimates hold:

$$
\begin{aligned}
\|S(q)\|_{V \times P} & \leq c, \\
\left\|S^{\prime}(q)(\delta q)\right\|_{V \times P} & \leq c\|\delta q\|_{H^{2}(I)}, \\
\left\|S^{\prime \prime}(q)(\delta q, \delta q)\right\|_{V \times P} & \leq c\|\delta q\|_{H^{2}(I)}^{2},
\end{aligned}
$$

provided that the data satisfy the following regularity requirements:

$$
\eta, \nu \in W^{2, \infty}(\widehat{\Omega}), \quad \mathbf{f} \in\left[H^{1}(\widehat{\Omega})\right]^{2} .
$$

Proof. We preliminary note that problems (3.2)-(3.3) have the same form of (2.8), but with different righthand sides. Therefore, the thesis can be proved in the same way as in Proposition 2.7. The only extra result to be shown, similarly to (2.10d), is the continuity of the right-hand sides in (3.2)-(3.3). This is easily achieved resorting to Proposition 3.4, which also makes the dependence on $\|\delta q\|_{H^{2}(I)}$ explicit.

Indeed, under the regularity assumptions on the data functions and employing Hölder inequality and the boundedness of the coefficients (see Prop. 3.4, points 1-5) we get the following estimates, for any $q \in Q^{a d}$, $\delta q \in \delta Q, \mathbf{u}, \mathbf{v} \in V, \pi \in P:$

$$
\begin{aligned}
\dot{a}(q, \delta q)(\mathbf{u}, \mathbf{v}) & \leq \dot{M}\|\delta q\|_{H^{2}(I)}\|\nabla \mathbf{u}\|\|\nabla \mathbf{v}\| \\
\dot{b}(q, \delta q)(\mathbf{v}, \pi) & \leq c\|\delta q\|_{H^{2}(I)}\|\nabla \mathbf{v}\|\|\pi\| \\
\dot{F}(q, \delta q)(\mathbf{v}) & \leq\left(c\|\mathbf{f}\|_{\left[H^{1}(\widehat{\Omega})\right]^{2}}\|\delta q\|_{H^{2}(I)}+\dot{M} c\left\|\mathbf{g}_{D}\right\|_{\left[H^{1 / 2}\left(\Gamma_{3}\right)\right]^{2}}\|\delta q\|_{H^{2}(I)}\right)\|\nabla \mathbf{v}\| \\
\dot{G}(q, \delta q)(\pi) & \leq c\|\delta q\|_{H^{2}(I)} c_{\mathcal{R}}\left\|\mathbf{g}_{D}\right\|_{\left[H^{1 / 2}\left(\Gamma_{3}\right)\right]^{2}}\|\pi\|,
\end{aligned}
$$

where $\dot{M}=c_{1}\|\eta\|_{W^{1, \infty}}+c_{2}\|\nu\|_{W^{1, \infty}}$, being $c, c_{1}, c_{2}$ constants that are independent of $q, \delta q, \mathbf{u}, \mathbf{v}, \pi$. Similar estimates hold for the twice-dotted forms:

$$
\begin{gathered}
\ddot{a}(q, \delta q, \delta q)(\mathbf{u}, \mathbf{v}) \leq c\|\delta q\|_{H^{2}(I)}^{2}\|\nabla \mathbf{u}\|\|\nabla \mathbf{v}\| \\
\ddot{b}(q, \delta q, \delta q)(\mathbf{v}, \pi) \leq c\|\delta q\|_{H^{2}(I)}^{2}\|\nabla \mathbf{v}\|\|\pi\| \\
\ddot{F}(q, \delta q, \delta q)(\mathbf{v}) \leq c\|\delta q\|_{H^{2}(I)}^{2}\|\nabla \mathbf{v}\| \\
\ddot{G}(q, \delta q, \delta q)(\pi) \leq c\|\delta q\|_{H^{2}(I)}^{2}\|\pi\| .
\end{gathered}
$$

Collecting all the above estimates, both the right-hand sides of problems (3.2)-(3.3) result to be continuous. Finally, applying the results on saddle-point problems in [18] yields the thesis. 
Hinging upon the problems (3.2)-(3.3) defining the first and second derivatives of the solution w.r.t. the control, we are now ready to compute the derivatives of $j$, as follows:

$$
\begin{aligned}
j(q)= & \left(\nabla \mathbf{u} A_{q}, \nabla \mathbf{u}\right)+\frac{\alpha}{2}\left\|q^{\prime \prime}\right\|_{I}^{2}+\frac{\beta}{2}\left(\int_{I} q(x) \mathrm{d} x-\bar{V}\right)^{2}, \\
j^{\prime}(q)(\delta q)= & \left(\nabla \mathbf{u} A_{q, \delta q}^{\prime}, \nabla \mathbf{u}\right)+2\left(\nabla \delta \mathbf{u} A_{q}, \nabla \mathbf{u}\right)+\alpha\left(\delta q^{\prime \prime}, q^{\prime \prime}\right)_{I} \\
& +\beta\left(\int_{I} q(x) \mathrm{d} x-\bar{V}\right) \int_{I} \delta q(x) \mathrm{d} x, \\
j^{\prime \prime}(q)(\delta q, \tau q)= & \left(\nabla \mathbf{u} A_{q, \delta q, \tau q}^{\prime \prime}, \nabla \mathbf{u}\right)+2\left(\nabla \tau \mathbf{u} A_{q, \delta q}^{\prime}+\nabla \delta \mathbf{u} A_{q, \tau q}^{\prime}, \nabla \mathbf{u}\right) \\
+ & 2\left(\nabla \delta \mathbf{u} A_{q}, \nabla \tau \mathbf{u}\right)+2\left(\nabla \tau \delta \mathbf{u} A_{q}, \nabla \mathbf{u}\right) \\
+ & \alpha\left(\delta q^{\prime \prime}, \tau q^{\prime \prime}\right)_{I}+\beta \int_{I} \delta q(x) \mathrm{d} x \int_{I} \tau q(x) \mathrm{d} x,
\end{aligned}
$$

where $\mathbf{u}, \delta \mathbf{u}, \tau \mathbf{u}, \tau \delta \mathbf{u}$ are the same as in (3.2) and (3.3). The continuity of the derivatives is an easy consequence of the regularity and symmetry of the matrix $A_{q}$ and its derivatives.

The expressions (3.4b) and (3.4c) for the first and second derivatives of the functional $j$ have been derived employing the usual differentiation rules; the general structure for the derivatives of integral shape functionals can be found, e.g., in [25].

\subsection{Control discretization}

Let $\left\{I_{i}=\left(x_{i-1}, x_{i}\right)\right\}_{i=1}^{N}$ be a partition of the domain $I$, with discretization parameter $\sigma=\max _{i \in\{1, \ldots, N\}}\left|I_{i}\right|$. We can then define the discrete controls set as

$$
Q_{\sigma}^{a d}=Q^{a d} \cap Q_{\sigma}, \text { with } Q_{\sigma}=\left\{q \in C^{0}(\bar{I}):\left.q\right|_{I_{i}} \in \mathbb{P}_{4}\left(I_{i}\right), i \in\{1, \ldots, N\}\right\} .
$$

The semi-discretized optimization problem reads as follows

$$
\min _{q_{\sigma} \in Q_{\sigma}^{a d}} j\left(q_{\sigma}\right)=\min _{q_{\sigma} \in Q_{\sigma}^{a d}} J\left(q_{\sigma}, S\left(q_{\sigma}\right) \circ T_{q_{\sigma}}^{-1}\right) .
$$

As $Q_{\sigma}^{a d} \subseteq Q^{a d}$, the minimization problem (3.5) inherits the existence and regularity properties holding for the original continuous optimization problem (2.9).

Let us denote by $\Pi_{\sigma}^{4}: L^{2}(I) \rightarrow Q_{\sigma}$ the classical polynomial interpolation operator and notice that $\Pi_{\sigma}^{4}\left(Q^{a d}\right) \subseteq$ $Q_{\sigma}^{a d}$. Standard interpolation error estimates hold (see, e.g., [7]): for $r \geq 1,0 \leq m \leq r+1$, it holds that

$$
\left|q-\Pi_{\sigma}^{r} q\right|_{H^{m}(I)} \leq c \sigma^{r+1-m}|q|_{H^{r+1}(I)} \quad \forall q \in H^{r+1}(I) .
$$

In this section, we aim at proving the following convergence result:

Proposition 3.6. Let $\bar{q} \in Q^{a d}$ be the exact solution of (2.9), and $\bar{q}_{\sigma}$ the solution of the partially discretized problem (3.5). Then, assuming that the optimal control $\bar{q}$ belongs to $H^{5}(I)$, the following convergence error estimate holds:

$$
\left\|\bar{q}-\bar{q}_{\sigma}\right\|_{H^{3}(I)} \leq c \sigma^{2}|\bar{q}|_{H^{5}(I)} .
$$

We observe that Proposition 3.6 needs the optimal control $\bar{q}$ to belong to $H^{5}(I)$. To achieve this regularity, there is no need to re-define the admissible controls set $Q^{a d}$, but it is sufficient to assume the validity of the following regularity result for the classical Stokes problem. 
Assumption 3.7. Let $\Omega_{q}$ be an open, bounded set of $\mathbb{R}^{2}$ and let $\Gamma_{q}$ be $C^{1,1}$ and $\partial \Omega_{q} \backslash \Gamma_{q}$ polygonal, with $\partial \Omega_{q}$ having convex corners. Let us assume that data functions fulfill the following requests:

$$
\nu \in H^{3}(\widehat{\Omega}), \quad \eta \in H^{2}(\widehat{\Omega}), \quad \mathbf{f} \in\left[H^{2}(\widehat{\Omega})\right]^{2}, \quad \mathbf{g}_{D} \in\left[H^{5 / 2}\left(\Gamma_{3}\right)\right]^{2}, \quad \mathbf{g}_{N} \in\left[H^{3 / 2}\left(\Gamma_{1}\right)\right]^{2},
$$

and suitable compatibility conditions. Then, for the solution $(\widetilde{\mathbf{u}}, \widetilde{p})$ of $(2.4)$, the following hold:

(a) $(\widetilde{\mathbf{u}}, \widetilde{p}) \in\left[H^{3}\left(\Omega_{q}\right)\right]^{2} \times H^{2}\left(\Omega_{q}\right)$

(b) $\|\widetilde{\mathbf{u}}\|_{\left[H^{3}\left(\Omega_{q}\right)\right]^{2}}+\|\widetilde{p}\|_{H^{2}\left(\Omega_{q}\right)} \leq c\left(\eta, \nu, \mathbf{g}_{D}, \mathbf{g}_{N}, \mathbf{f}, \widehat{\Omega}\right)$.

Remark 3.8. Assumption 3.7 might be proved by resorting to results similar to those presented in [21].

The proof that Assumption 3.7 implies the $H^{5}$-regularity of the optimal control is reported in Appendix A: see Theorem A.4.

In order to prove Proposition 3.6, we need to collect some preliminary results that will be derived under the following two assumptions, already employed in [26].

Assumption 3.9 ([26], Assumption 1.5). For the optimal solution $\bar{q}$ of problem (2.9), the constraint $q \leq 1-\varepsilon$ is not active, i.e.

$$
\exists \delta>0 \text { such that } \bar{q}(x) \leq 1-\varepsilon-\delta \quad \forall x \in I .
$$

Assumption 3.10 ([26], Assumption 3.1). For any local minimum $\bar{q}$, we have

$$
j^{\prime \prime}(\bar{q})(\delta q, \delta q)>0 \quad \forall \delta q \in \delta Q \backslash\{0\} .
$$

We start by proving some regularity results on the solution operator $S$ and its derivatives.

Lemma 3.11. Let $S$ be the solution operator of the transformed Stokes problem (2.8). If there exists some $k>0$ such that

$$
\eta, \nu \in C^{k}(\overline{\widehat{\Omega}}), \quad \mathbf{f} \in\left[C^{k}(\overline{\widehat{\Omega}})\right]^{2},
$$

then $S$ is at least $k$ times continuously Fréchet differentiable.

Proof. The proof is the same as in Corollary 3.2, simply applying the Implicit Function Theorem in the form presented in Theorem 3.1.

Based on the previous result, we can prove the following:

Lemma 3.12. Let $k \in \mathbb{N}$ and

$$
\eta, \nu \in C^{k+1}(\overline{\widehat{\Omega}}), \quad \mathbf{f} \in\left[C^{k+1}(\overline{\widehat{\Omega}})\right]^{2},
$$

then, for any $q, r \in Q^{a d}$ and $\delta q_{1}, \delta q_{2}, \ldots, \delta q_{k} \in \delta Q$, the following inequalities hold:

$$
\left\|S^{(i)}(q)\left(\delta q_{1}, \ldots, \delta q_{i}\right)-S^{(i)}(r)\left(\delta q_{1}, \ldots, \delta q_{i}\right)\right\|_{V \times P} \leq c\|q-r\|_{H^{2}(I)} \prod_{j=1}^{i}\left\|\delta q_{j}\right\|_{H^{2}(I)}, \quad \text { for } i=0, \ldots, k .
$$


Proof. Let $q$ and $r$ be two control functions in $Q^{a d}$ and $\delta q_{1}, \ldots, \delta q_{k}$ admissible control variations. Applying Lemma 3.11 under the hypotheses of the present lemma, we get

$$
S \in C^{k+1}\left(\operatorname{int}\left(Q^{a d}\right) ; V \times P\right) .
$$

Let us consider $k=0$. As $S \in C^{1}$, given the control functions $q, r \in Q^{a d}$, the Mean Value Theorem ensures that

$$
\exists \xi \in Q^{a d} \text { such that } S(q)-S(r)=S^{\prime}(\xi)(q-r) .
$$

Being the Fréchet derivative $S^{\prime}(\xi)$ a linear operator on the control variation, its continuity is equivalent to its boundedness, thus we get

$$
\|S(q)-S(r)\|_{V \times P}=\left\|S^{\prime}(\xi)(q-r)\right\|_{V \times P} \leq c\|q-r\|_{H^{2}(I)} .
$$

We remark that $c$ does not depend on $q, r$, even though $\xi$ does: indeed, $\left\|S^{\prime}(\xi)\right\|_{\mathscr{L}(\delta Q ; V \times P)}=$ $\sup _{\delta q \in \delta Q:\|\delta q\|_{H^{3}(I)}=1}\left\|S^{\prime}(\xi)(\delta q)\right\|_{V \times P}$, which is upper bounded by a constant depending only on the data of the state problem, thanks to Theorem 3.5.

In the general case $k>0$, for each $i \in\{0, \ldots, k\}$ there exists $\xi_{i} \in Q^{a d}$ such that

$$
S^{(i)}(q)-S^{(i)}(r)=S^{(i+1)}\left(\xi_{i}\right)(q-r),
$$

where we remark that (3.7) is an equality between linear operators belonging to $\mathscr{L}_{i}:=\mathscr{L}\left(\delta Q^{i} ; V \times P\right)$. Observing that $S^{(i+1)}\left(\xi_{i}\right) \in \mathscr{L}_{i+1}$, we can proceed as before to obtain

$$
\begin{aligned}
& \left\|S^{(i)}(q)\left(\delta q_{1}, \ldots, \delta q_{i}\right)-S^{(i)}(r)\left(\delta q_{1}, \ldots, \delta q_{i}\right)\right\|_{V \times P} \\
& \leq\left\|S^{(i)}(q)-S^{(i)}(r)\right\| \mathscr{L}_{i} \prod_{j=1}^{i}\left\|\delta q_{j}\right\|_{H^{2}(I)}=\left\|S^{(i+1)}\left(\xi_{i}\right)(q-r)\right\|_{\mathscr{L}_{i}} \prod_{j=1}^{i}\left\|\delta q_{j}\right\|_{H^{2}(I)} \\
& \leq\left\|S^{(i+1)}\left(\xi_{i}\right)\right\| \mathscr{L}_{i+1}\|q-r\|_{H^{2}(I)} \prod_{j=1}^{i}\left\|\delta q_{j}\right\|_{H^{2}(I)} .
\end{aligned}
$$

Finally, employing a similar result to Theorem $3.5,\left\|S^{(i+1)}(\xi)\right\|_{\mathscr{L}_{i+1}}$ can be bounded by a constant $c>0$, uniformly on $\xi \in Q^{a d}$. Hence the proof is complete.

The continuity of the solution operator $S$ directly implies the continuity of the functional $j$, as stated in the following result.

Lemma 3.13. For any $q, r \in Q^{a d}$ and any $\delta q \in \delta Q$, it holds that

(a) $|j(q)-j(r)| \leq c\|q-r\|_{H^{2}(I)}$,

(b) $\left|j^{\prime}(q)(\delta q)-j^{\prime}(r)(\delta q)\right| \leq c\|q-r\|_{H^{2}(I)}\|\delta q\|_{H^{2}(I)}$,

(c) $\left|j^{\prime \prime}(q)(\delta q, \delta q)-j^{\prime \prime}(r)(\delta q, \delta q)\right| \leq c\|q-r\|_{H^{2}(I)}\|\delta q\|_{H^{2}(I)}^{2}$.

Proof. Let us fix $q, r \in Q^{a d}$. To simplify the notation, let $S(q)=(\mathbf{u}, p)$ and $S(r)=(\mathbf{z}, s)$. As the proofs of (a) - (c) are similar, we focus on (c), highlighting the most technical parts.

Bearing in mind the expression of $j^{\prime \prime}$ (see (3.4c)), we first focus on the following term:

$$
\begin{aligned}
& \left|\left(\nabla \mathbf{u} A_{q, \delta q, \delta q}^{\prime \prime}, \nabla \mathbf{u}\right)-\left(\nabla \mathbf{z} A_{r, \delta q, \delta q}^{\prime \prime}, \nabla \mathbf{z}\right)\right| \\
& =\left|\left((\nabla \mathbf{u}-\nabla \mathbf{z}) A_{q, \delta q, \delta q}^{\prime \prime}, \nabla \mathbf{u}+\nabla \mathbf{z}\right)+\left(\nabla \mathbf{z}\left(A_{q, \delta q, \delta q}^{\prime \prime}-A_{r, \delta q, \delta q}^{\prime \prime}\right), \nabla \mathbf{z}\right)\right| \\
& \leq\left\|A_{q, \delta q, \delta q}^{\prime \prime}\right\|_{\infty}(\|\nabla \mathbf{u}\|+\|\nabla \mathbf{z}\|)\|\nabla \mathbf{u}-\nabla \mathbf{z}\|+\|\nabla \mathbf{z}\|^{2}\left\|A_{q, \delta q, \delta q}^{\prime \prime}-A_{r, \delta q, \delta q}^{\prime \prime}\right\|_{\infty} \\
& \leq c\|q-r\|_{H^{2}(I)}\|\delta q\|_{H^{2}(I)}^{2},
\end{aligned}
$$


where the state variables have been bounded using Proposition 2.7 and Lemma 3.12, while the terms involving $A_{q, \delta q, \delta q}^{\prime \prime}, A_{r, \delta q, \delta q}^{\prime \prime}$ has been handed employing Proposition 3.4.

Using the same results, it is easy to bound also the following term:

$$
\begin{aligned}
& \left|\left(\nabla \delta \mathbf{u} A_{q, \delta q}^{\prime}, \nabla \mathbf{u}\right)-\left(\nabla \delta \mathbf{z} A_{r, \delta q}^{\prime}, \nabla \mathbf{z}\right)\right| \\
& =\left|\left((\nabla \delta \mathbf{u}-\nabla \delta \mathbf{z}) A_{q, \delta q}^{\prime}, \nabla \mathbf{u}\right)+\left(\nabla \delta \mathbf{z}\left(A_{q, \delta q}^{\prime}-A_{r, \delta q}^{\prime}\right), \nabla \mathbf{u}\right)+\left(\nabla \delta \mathbf{z} A_{r, \delta q}^{\prime}, \nabla \mathbf{u}-\nabla \mathbf{z}\right)\right| .
\end{aligned}
$$

All the other terms entering in $j^{\prime \prime}$ can be treated in a similar way, to get (c).

The results stated so far are sufficient to prove the following coercivity result on $j$.

Lemma 3.14. If $\bar{q}$ is a local solution of (2.9), fulfilling Assumption 3.10, then there exist $\delta_{1}, \delta_{2}>0$ such that, if $\|\bar{q}-r\|_{H^{2}(I)} \leq \delta_{1}$ for $r \in Q^{a d}$, then

$$
j^{\prime \prime}(r)(\delta q, \delta q) \geq \frac{\delta_{2}}{2}\|\delta q\|_{H^{3}(I)}^{2} \quad \forall \delta q \in \delta Q .
$$

Proof. Following the ideas of ([26], Thm. 3.13, Lem. 3.14), let us begin by proving by contradiction that inequality (3.8) holds for $r=\bar{q}$. Assuming that it does not hold, there should exist a sequence $\left\{\delta q_{n}\right\}_{n \in \mathbb{N}} \subset \delta Q$ such that $\left\|\delta q_{n}\right\|_{H^{3}(I)}=1$ for any $n \in \mathbb{N}$ and

$$
j^{\prime \prime}(\bar{q})\left(\delta q_{n}, \delta q_{n}\right)<\frac{1}{n} .
$$

Being the sequence $\left\{\delta q_{n}\right\}$ bounded, it has an accumulation point $\overline{\delta q}$ in $H^{3}(I)$, and thanks to the second order optimality condition $j^{\prime \prime}(\bar{q})(\delta q, \delta q) \geq 0$ and to the lower semicontinuity of the functional $\delta q \mapsto j^{\prime \prime}(\bar{q})(\delta q, \delta q)$ (whose proof can be found in Cor. B.2, in the Appendix), we have that

$$
0 \leq j^{\prime \prime}(\bar{q})(\overline{\delta q}, \overline{\delta q}) \leq \liminf _{n \rightarrow \infty} j^{\prime \prime}(\bar{q})\left(\delta q_{n}, \delta q_{n}\right) \leq \limsup _{n \rightarrow \infty} j^{\prime \prime}(\bar{q})\left(\delta q_{n}, \delta q_{n}\right) \leq \lim _{n \rightarrow \infty} \frac{1}{n}=0 .
$$

Therefore, the whole sequence satisfies $j^{\prime \prime}(\bar{q})\left(\delta q_{n}, \delta q_{n}\right) \rightarrow j^{\prime \prime}(\bar{q})(\overline{\delta q}, \overline{\delta q})=0$. Thus, Assumption 3.10 would imply $\overline{\delta q}=0$, which is impossible, since $\left\|\delta q_{n}\right\|_{H^{3}(I)}=1 \forall n \in \mathbb{N}$.

Having proved inequality (3.8) for $r=\bar{q}$, the result can be easily extended to a whole neighborhood of $\bar{q}$ by simple calculations and exploiting Lemma 3.13(c).

Now, we are ready to conclude this section with the proof of Proposition 3.6.

Proof of Proposition 3.6. For sufficiently small $\sigma$, the Mean Value Theorem and Lemma 3.14 imply the existence of some $t \in[0,1]$ such that, for $\xi=t \Pi_{\sigma}^{4} \bar{q}+(1-t) \bar{q}_{\sigma}$, we have

$$
\begin{aligned}
\frac{\delta_{2}}{2}\left\|\Pi_{\sigma}^{4} \bar{q}-\bar{q}_{\sigma}\right\|_{H^{3}(I)}^{2} & \leq j^{\prime \prime}(\xi)\left(\Pi_{\sigma}^{4} \bar{q}-\bar{q}_{\sigma}, \Pi_{\sigma}^{4} \bar{q}-\bar{q}_{\sigma}\right) \\
& =j^{\prime}\left(\Pi_{\sigma}^{4} \bar{q}\right)\left(\Pi_{\sigma}^{4} \bar{q}-\bar{q}_{\sigma}\right)-j^{\prime}\left(\bar{q}_{\sigma}\right)\left(\Pi_{\sigma}^{4} \bar{q}-\bar{q}_{\sigma}\right) \\
& \stackrel{\mathrm{a}}{=} j^{\prime}\left(\Pi_{\sigma}^{4} \bar{q}\right)\left(\Pi_{\sigma}^{4} \bar{q}-\bar{q}_{\sigma}\right)-j^{\prime}(\bar{q})\left(\Pi_{\sigma}^{4} \bar{q}-\bar{q}_{\sigma}\right) \\
& \stackrel{\mathrm{b}}{\leq} c\left\|\bar{q}-\Pi_{\sigma}^{4} \bar{q}\right\|_{H^{3}(I)}\left\|\Pi_{\sigma}^{4} \bar{q}-\bar{q}_{\sigma}\right\|_{H^{3}(I)} \\
& \stackrel{\mathrm{c}}{\leq} c \sigma^{2}\|\bar{q}\|_{H^{5}(I)}\left\|\Pi_{\sigma}^{4} \bar{q}-\bar{q}_{\sigma}\right\|_{H^{3}(I)},
\end{aligned}
$$


where we used:

(a) $j^{\prime}(\bar{q})\left(\Pi_{\sigma}^{4} \bar{q}-\bar{q}_{\sigma}\right)=j^{\prime}\left(\bar{q}_{\sigma}\right)\left(\Pi_{\sigma}^{4} \bar{q}-\bar{q}_{\sigma}\right)=0$, due to Assumption 3.9 and then the first order optimality condition;

(b) point b of Lemma 3.13 and the fact that $\|\cdot\|_{H^{2}(I)} \leq\|\cdot\|_{H^{3}(I)}$;

(c) the interpolation error estimate (3.6).

From (3.9), we obtain

$$
\left\|\Pi_{\sigma}^{4} \bar{q}-\bar{q}_{\sigma}\right\|_{H^{3}(I)} \leq c \sigma^{2}\|\bar{q}\|_{H^{5}(I)} .
$$

Finally, triangular inequality gives the thesis.

\subsection{State discretization}

Let $\mathcal{T}_{h}$ be a regular triangulation of $\Omega_{0}$, with discretization parameter $h=\max _{K \in \mathcal{T}_{h}}|K|$. We can thus introduce the finite element spaces

$$
\begin{aligned}
X_{h}^{r}\left(\Omega_{0}\right) & =\left\{\varphi \in C^{0}\left(\bar{\Omega}_{0}\right):\left.\varphi\right|_{K} \in \mathbb{P}_{r}(K) \quad \forall K \in \mathcal{T}_{h}\right\} \\
V_{h} & =V \cap\left[X_{h}^{2}\left(\Omega_{0}\right)\right]^{2}, \\
P_{h} & =P \cap X_{h}^{1}\left(\Omega_{0}\right),
\end{aligned}
$$

where $\mathbb{P}_{r}(K)$ is the space of polynomials on $K$ having degree less than or equal to $r$.

Passing from the continuous to the discrete case, the variational forms involved in problem (2.8) preserve all their properties, with the discrete inf-sup condition ensured by the following:

Proposition 3.15. There exists a positive constant $\bar{\beta}$ such that

$$
\forall \pi_{h} \in P_{h} \exists \mathbf{v}_{h} \in V_{h}: b(q)\left(\mathbf{v}_{h}, \pi_{h}\right) \geq \bar{\beta}\left\|\nabla \mathbf{v}_{h}\right\|\left\|\pi_{h}\right\|,
$$

and $\bar{\beta}$ is independent of $q \in Q^{a d}$ and of $h \in[0, \widehat{h}]$, for a certain $\widehat{h}>0$.

Proof. From FEM approximation of Stokes problem [18], we know that pair $\left(V_{h}, P_{h}\right)$ is stable, i.e. there exists a constant $\widehat{\beta}>0$ such that

$$
\forall \pi_{h} \in P_{h} \exists \mathbf{v}_{h} \in V_{h}: b(0)\left(\mathbf{v}_{h}, \pi_{h}\right) \geq \widehat{\beta}\left\|\nabla \mathbf{v}_{h}\right\|\left\|\pi_{h}\right\|,
$$

with $\widehat{\beta}$ independent of $h \in[0, \widehat{h}]$.

In order to show that such discrete spaces fulfill inf-sup condition also for the transformed form $b(q)$, one can just follow the steps presented in Section 2.2, with constant $\widehat{\beta}$ from (3.12). Indeed, no assumptions on the spaces $V, P$ have been made there, apart from the validity of the inf-sup condition for $b(0)$.

The finite element discretization of (2.8) reads as follows:

$$
\begin{aligned}
& \text { Find }\left(\mathbf{u}_{h}, p_{h}\right) \in V_{h} \times P_{h}, \text { such that } \\
& \left\{\begin{aligned}
a\left(q_{\sigma}\right)\left(\mathbf{u}_{h}, \mathbf{v}_{h}\right)+b\left(q_{\sigma}\right)\left(\mathbf{v}_{h}, p_{h}\right)=F\left(q_{\sigma}\right)\left(\mathbf{v}_{h}\right) & \forall \mathbf{v}_{h} \in V_{h}, \\
b\left(q_{\sigma}\right)\left(\mathbf{u}_{h}, \pi_{h}\right)=G\left(q_{\sigma}\right)\left(\pi_{h}\right) & \forall \pi_{h} \in P_{h} .
\end{aligned}\right.
\end{aligned}
$$

The well-posedness of (3.13) stems from the validity of (3.11).

The discrete state solution operator, resulting from problem (3.13), and the corresponding discrete cost functional are defined as

$$
S_{h}: Q_{\sigma}^{a d} \rightarrow V_{h} \times P_{h}, \text { with } S_{h}\left(q_{\sigma}\right)=\left(\mathbf{u}_{h}, p_{h}\right), \quad j_{h}: Q_{\sigma}^{a d} \rightarrow \mathbb{R}, \text { with } j\left(q_{\sigma}\right)=J\left(q_{\sigma}, S_{h}\left(q_{\sigma}\right) \circ T_{q_{\sigma}}^{-1}\right),
$$

whereas the fully discretized shape optimization problem can be written as

$$
\min _{q_{\sigma} \in Q_{\sigma}^{a d}} j_{h}\left(q_{\sigma}\right):=\min _{q_{\sigma} \in Q_{\sigma}^{a d}} J\left(q_{\sigma}, S_{h}\left(q_{\sigma}\right) \circ T_{q_{\sigma}}^{-1}\right) .
$$


For future use, it is useful to explicitly write the problems defining the derivatives of $S_{h}$ :

(1) $S_{h}^{\prime}\left(q_{\sigma}\right)\left(\delta q_{\sigma}\right)=\left(\delta \mathbf{u}_{h}, \delta p_{h}\right) \in V_{h} \times P_{h}$, where $\left(\delta \mathbf{u}_{h}, \delta p_{h}\right)$ is the solution of

$$
\left\{\begin{array}{cc}
a\left(q_{\sigma}\right)\left(\delta \mathbf{u}_{h}, \mathbf{v}_{h}\right)+b\left(q_{\sigma}\right)\left(\mathbf{v}_{h}, \delta p_{h}\right)=\dot{F}\left(q_{\sigma}, \delta q_{\sigma}\right)\left(\mathbf{v}_{h}\right) & \\
-\dot{a}\left(q_{\sigma}, \delta q_{\sigma}\right)\left(\mathbf{u}_{h}, \mathbf{v}_{h}\right)-\dot{b}\left(q_{\sigma}, \delta q_{\sigma}\right)\left(\mathbf{v}_{h}, p_{h}\right) & \forall \mathbf{v}_{h} \in V_{h}, \\
b\left(q_{\sigma}\right)\left(\delta \mathbf{u}_{h}, \pi_{h}\right)=\dot{G}\left(q_{\sigma}, \delta q_{\sigma}\right)\left(\pi_{h}\right)-\dot{b}\left(q_{\sigma}, \delta q_{\sigma}\right)\left(\mathbf{u}_{h}, \pi_{h}\right) & \forall \pi_{h} \in P_{h} .
\end{array}\right.
$$

(2) $S_{h}^{\prime \prime}\left(q_{\sigma}\right)\left(\delta q_{\sigma}, \tau q_{\sigma}\right)=\left(\tau \delta \mathbf{u}_{h}, \tau \delta p_{h}\right) \in V_{h} \times P_{h}$, where $\left(\tau \delta \mathbf{u}_{h}, \tau \delta p_{h}\right)$ is the solution of

$$
\left\{\begin{aligned}
a\left(q_{\sigma}\right)\left(\tau \delta \mathbf{u}_{h}, \mathbf{v}_{h}\right)+b\left(q_{\sigma}\right)\left(\mathbf{v}_{h}, \tau \delta p_{h}\right)= & \\
\ddot{F}\left(q_{\sigma}, \delta q_{\sigma}, \tau q_{\sigma}\right)\left(\mathbf{v}_{h}\right) & \\
-\ddot{a}\left(q_{\sigma}, \delta q_{\sigma}, \tau q_{\sigma}\right)\left(\mathbf{u}_{h}, \mathbf{v}_{h}\right)-\ddot{b}\left(q_{\sigma}, \delta q_{\sigma}, \tau q_{\sigma}\right)\left(\mathbf{v}_{h}, p_{h}\right) & \\
-\dot{a}\left(q_{\sigma}, \delta q_{\sigma}\right)\left(\tau \mathbf{u}_{h}, \mathbf{v}_{h}\right)-\dot{b}\left(q_{\sigma}, \delta q_{\sigma}\right)\left(\mathbf{v}_{h}, \tau p_{h}\right) & \\
-\dot{a}\left(q_{\sigma}, \tau q_{\sigma}\right)\left(\delta \mathbf{u}_{h}, \mathbf{v}_{h}\right)-\dot{b}\left(q_{\sigma}, \tau q_{\sigma}\right)\left(\mathbf{v}_{h}, \delta p_{h}\right) & \\
b\left(q_{\sigma}\right)\left(\tau \delta \mathbf{u}_{h}, \pi_{h}\right)=\ddot{G}\left(q_{\sigma}, \delta q_{\sigma}, \tau q_{\sigma}\right)\left(\pi_{h}\right)-\ddot{b}\left(q_{\sigma}, \delta q_{\sigma}, \tau q_{\sigma}\right)\left(\mathbf{u}_{h}, \pi_{h}\right) & \\
-\dot{b}\left(q_{\sigma}, \delta q_{\sigma}\right)\left(\tau \mathbf{u}_{h}, \pi_{h}\right)-\dot{b}\left(q_{\sigma}, \tau q_{\sigma}\right)\left(\delta \mathbf{u}_{h}, \pi_{h}\right) & \forall \pi_{h} \in P_{h},
\end{aligned}\right.
$$

with $\left(\tau \mathbf{u}_{h}, \tau p_{h}\right)=S_{h}^{\prime}\left(q_{\sigma}\right)\left(\tau q_{\sigma}\right)$.

Like in the previous section, in order to study the convergence of the discrete quantities to their continuous counterparts, we introduce projection operators onto the discrete spaces. Since there will be no room for misunderstanding, to avoid redundant notation, all of them will be indicated by the same symbol $\Pi_{h}^{r}$, never minding if returning functions in $V_{h}, P_{h}$, or $V_{h} \times P_{h}$.

Referring to $X_{h}^{r}$, the following interpolation estimate is known (see e.g. [34], Sect. 3.4.2), for $r \geq 1, m=0,1$ :

$$
\left|\varphi-\Pi_{h}^{r} \varphi\right|_{H^{m}\left(\Omega_{0}\right)} \leq c h^{r+1-m}|\varphi|_{H^{r+1}\left(\Omega_{0}\right)} .
$$

The particular choice of $\mathbb{P}_{2}-\mathbb{P}_{1}$ couple in the spaces defined in (3.10) leads us to assume the following regularity on the state variables and their shape derivatives:

Assumption 3.16. For any $q \in Q^{a d}, \delta q \in \delta Q$, each of $S(q), S^{\prime}(q)(\delta q), S^{\prime \prime}(q)(\delta q, \delta q)$ belongs to $\left[H^{3}\left(\Omega_{0}\right)\right]^{2} \times$ $H^{2}\left(\Omega_{0}\right)$ and the following inequalities hold:

$$
\begin{aligned}
& \|S(q)\|_{\left[H^{3}\left(\Omega_{0}\right)\right]^{2} \times H^{2}\left(\Omega_{0}\right)}=\quad\|\mathbf{u}\|_{\left[H^{3}\left(\Omega_{0}\right)\right]^{2}}+\|p\|_{H^{2}\left(\Omega_{0}\right)} \leq c_{1}, \\
& \left\|S^{\prime}(q)(\delta q)\right\|_{\left[H^{3}\left(\Omega_{0}\right)\right]^{2} \times H^{2}\left(\Omega_{0}\right)}=\|\delta \mathbf{u}\|_{\left[H^{3}\left(\Omega_{0}\right)\right]^{2}}+\|\delta p\|_{H^{2}\left(\Omega_{0}\right)} \leq c_{2}\|\delta q\|_{H^{3}(I)}, \\
& \left\|S^{\prime \prime}(q)(\delta q, \delta q)\right\|_{\left[H^{3}\left(\Omega_{0}\right)\right]^{2} \times H^{2}\left(\Omega_{0}\right)}=\|\delta \delta \mathbf{u}\|_{\left[H^{3}\left(\Omega_{0}\right)\right]^{2}}+\|\delta \delta p\|_{H^{2}\left(\Omega_{0}\right)} \leq c_{3}\|\delta q\|_{H^{3}(I)}^{2} .
\end{aligned}
$$

Remark 3.17. In Appendix A we prove (Thm. A.3) the validity of Assumption 3.16, that involves suitable regularity assumptions on the data of the Stokes problem.

Assumption 3.16, together with (3.16), yields the following estimate:

$$
\begin{array}{cl}
\left\|\mathbf{u}-\Pi_{h}^{2} \mathbf{u}\right\|_{V}+\left\|p-\Pi_{h}^{1} p\right\|_{P} & \leq c_{1} h^{2}, \\
\left\|\delta \mathbf{u}-\Pi_{h}^{2} \delta \mathbf{u}\right\|_{V}+\left\|\delta p-\Pi_{h}^{1} \delta p\right\|_{P} & \leq c_{2} h^{2}\|\delta q\|_{H^{3}(I)}, \\
\left\|\delta \delta \mathbf{u}-\Pi_{h}^{2} \delta \delta \mathbf{u}\right\|_{V}+\left\|\delta \delta p-\Pi_{h}^{1} \delta \delta p\right\|_{P} & \leq c_{3} h^{2}\|\delta q\|_{H^{3}(I)}^{2} .
\end{array}
$$

Remark 3.18. Under regularity Assumption 3.16 , one can afford the optimal convergence rate for $\mathbb{P}_{2}-\mathbb{P}_{1}$ discretization: lower regularity of the state variables would lead to a lower convergence rate in (3.17). 
The interpolation error estimates are once again the basis upon which we build our convergence result, which reads as follows:

Lemma 3.19. For any $q_{\sigma} \in Q_{\sigma}^{a d}, \delta q_{\sigma} \in \delta Q_{\sigma}=\delta Q \cap Q_{\sigma}$, the following convergence estimates hold:

(a) $\left\|S\left(q_{\sigma}\right)-S_{h}\left(q_{\sigma}\right)\right\|_{V \times P} \leq c h^{2}$;

(b) $\left\|S^{\prime}\left(q_{\sigma}\right)\left(\delta q_{\sigma}\right)-S_{h}^{\prime}\left(q_{\sigma}\right)\left(\delta q_{\sigma}\right)\right\|_{V \times P} \leq c h^{2}\left\|\delta q_{\sigma}\right\|_{H^{3}(I)}$;

(c) $\left\|S^{\prime \prime}\left(q_{\sigma}\right)\left(\delta q_{\sigma}, \delta q_{\sigma}\right)-S_{h}^{\prime \prime}\left(q_{\sigma}\right)\left(\delta q_{\sigma}, \delta q_{\sigma}\right)\right\|_{V \times P} \leq c h^{2}\left\|\delta q_{\sigma}\right\|_{H^{3}(I)}^{2}$.

Proof. Since the discrete problems (3.13)-(3.15) fulfill the same properties as the continuous ones, we have that Theorem 3.5 on the boundedness of the continuous solution operator $S$ is true also for the discrete operator $S_{h}$ and its derivatives. Hinging upon this result and Assumption 3.16, we fix some $q_{\sigma} \in Q_{\sigma}^{a d}, \delta q_{\sigma} \in \delta Q_{\sigma}$ and proceed according to the following steps.

We first prove (a). From [18] and the independence of the continuity, coercivity and LBB constants from $q_{\sigma}$ and $h$, we can obtain the classical convergence result for a saddle-point problem, i.e.,

$$
\left\|S\left(q_{\sigma}\right)-S_{h}\left(q_{\sigma}\right)\right\|_{V \times P} \leq c\left(\left\|\mathbf{u}-\Pi_{h}^{2} \mathbf{u}\right\|_{V}+\left\|p-\Pi_{h}^{1} p\right\|_{P}\right) \leq c h^{2},
$$

with the last inequality exploiting interpolation error estimate (3.17).

We now proceed to prove (b). We set $(\mathbf{u}, p)=S\left(q_{\sigma}\right),(\delta \mathbf{u}, \delta p)=S^{\prime}\left(q_{\sigma}\right)\left(\delta q_{\sigma}\right),(\delta \delta \mathbf{u}, \delta \delta p)=S^{\prime \prime}\left(q_{\sigma}\right)\left(\delta q_{\sigma}, \delta q_{\sigma}\right)$, with subscript $\cdot_{h}$ denoting the corresponding discrete quantities, and we introduce the "intermediate derivative" $\left(\delta \widehat{\mathbf{u}}_{h}, \delta \widehat{p}_{h}\right)$, solution in $V_{h} \times P_{h}$ of the following problem: ${ }^{6}$

$$
\left\{\begin{array}{rlrl}
a\left(q_{\sigma}\right)\left(\delta \widehat{\mathbf{u}}_{h}, \mathbf{v}_{h}\right)+b\left(q_{\sigma}\right)\left(\mathbf{v}_{h}, \delta \widehat{p}_{h}\right)= & \dot{F}\left(q_{\sigma}, \delta q_{\sigma}\right)\left(\mathbf{v}_{h}\right) & \\
& -\dot{a}\left(q_{\sigma}, \delta q_{\sigma}\right)\left(\mathbf{u}, \mathbf{v}_{h}\right)-\dot{b}\left(q_{\sigma}, \delta q_{\sigma}\right)\left(\mathbf{v}_{h}, p\right) & & \forall \mathbf{v}_{h} \in V_{h}, \\
b\left(q_{\sigma}\right)\left(\delta \widehat{\mathbf{u}}_{h}, \pi_{h}\right) & =\dot{G}\left(q_{\sigma}, \delta q_{\sigma}\right)\left(\pi_{h}\right)-\dot{b}\left(q_{\sigma}, \delta q_{\sigma}\right)\left(\mathbf{u}_{h}, \pi_{h}\right) & & \forall \pi_{h} \in P_{h} .
\end{array}\right.
$$

Thanks to (3.18), we can separate the error due to the discretization of the problem on $S^{\prime}\left(q_{\sigma}\right)\left(\delta q_{\sigma}\right)$ from the one that is inherited from the discretization of $S\left(q_{\sigma}\right)$. Using triangular inequality yields

$$
\begin{aligned}
& \left\|S^{\prime}\left(q_{\sigma}\right)\left(\delta q_{\sigma}\right)-S_{h}^{\prime}\left(q_{\sigma}\right)\left(\delta q_{\sigma}\right)\right\|_{V \times P} \\
\leq & \left\|S^{\prime}\left(q_{\sigma}\right)\left(\delta q_{\sigma}\right)-\left(\delta \widehat{\mathbf{u}}_{h}, \delta \widehat{p}_{h}\right)\right\|_{V \times P}+\left\|\left(\delta \widehat{\mathbf{u}}_{h}, \delta \widehat{p}_{h}\right)-S_{h}^{\prime}\left(q_{\sigma}\right)\left(\delta q_{\sigma}\right)\right\|_{V \times P} \\
= & \left\|\delta \mathbf{u}-\delta \widehat{\mathbf{u}}_{h}\right\|_{V}+\left\|\delta \widehat{\mathbf{u}}_{h}-\delta \mathbf{u}_{h}\right\|_{V}+\left\|\delta p-\delta \widehat{p}_{h}\right\|_{P}+\left\|\delta \widehat{p}_{h}-\delta p_{h}\right\|_{P} .
\end{aligned}
$$

Considering the first term in (3.19), we have that, for any $\mathbf{w}_{h} \in V_{h}$,

$$
\begin{aligned}
& \alpha_{c}\left\|\nabla \delta \mathbf{u}-\nabla \delta \widehat{\mathbf{u}}_{h}\right\|^{2} \leq a\left(q_{\sigma}\right)\left(\delta \mathbf{u}-\delta \widehat{\mathbf{u}}_{h}, \delta \mathbf{u}-\delta \widehat{\mathbf{u}}_{h}\right) \\
= & a\left(q_{\sigma}\right)\left(\delta \mathbf{u}-\delta \widehat{\mathbf{u}}_{h}, \delta \mathbf{u}-\mathbf{w}_{h}\right)+a\left(q_{\sigma}\right)\left(\delta \mathbf{u}-\delta \widehat{\mathbf{u}}_{h}, \mathbf{w}_{h}-\delta \widehat{\mathbf{u}}_{h}\right) \\
= & a\left(q_{\sigma}\right)\left(\delta \mathbf{u}-\delta \widehat{\mathbf{u}}_{h}, \delta \mathbf{u}-\mathbf{w}_{h}\right)-b\left(q_{\sigma}\right)\left(\mathbf{w}_{h}-\delta \widehat{\mathbf{u}}_{h}, \delta p-\delta \widehat{p}_{h}\right),
\end{aligned}
$$

with the equality holding thanks to the fact that the first equations in (3.2) and (3.18) share the same right-hand side. Since (3.20) holds for every $\mathbf{w}_{h} \in V_{h}$, it still holds if we take the infimum w.r.t. $\mathbf{w}_{h}$. For the first term of the right-hand side we get

$$
\begin{aligned}
& \inf _{\mathbf{w}_{h} \in V_{h}} a\left(q_{\sigma}\right)\left(\delta \mathbf{u}-\delta \widehat{\mathbf{u}}_{h}, \delta \mathbf{u}-\mathbf{w}_{h}\right) \leq a\left(q_{\sigma}\right)\left(\delta \mathbf{u}-\delta \widehat{\mathbf{u}}_{h}, \delta \mathbf{u}-\Pi_{h}^{2} \delta \mathbf{u}\right) \\
& \leq M\left\|\nabla \delta \mathbf{u}-\nabla \delta \widehat{\mathbf{u}}_{h}\right\|\left\|\nabla \delta \mathbf{u}-\nabla \Pi_{h}^{2} \delta \mathbf{u}\right\| \leq c h^{2} M\left\|\nabla \delta \mathbf{u}-\nabla \delta \widehat{\mathbf{u}}_{h}\right\|\left\|\delta q_{\sigma}\right\|_{H^{3}(I)},
\end{aligned}
$$

where we employed the interpolation error estimate (3.17). Instead, taking $\mathbf{w}_{h}=\delta \widehat{\mathbf{u}}_{h}$ in the second term yields

$$
\inf _{\mathbf{w}_{h} \in V_{h}}\left[-b\left(q_{\sigma}\right)\left(\mathbf{w}_{h}-\delta \widehat{\mathbf{u}}_{h}, \delta p-\delta \widehat{p}_{h}\right)\right] \leq 0 .
$$

\footnotetext{
${ }^{6}$ The problem here introduced is a combination of problem $(3.2)$ for $S^{\prime}\left(q_{\sigma}\right)(\delta q)$ and its discrete counterpart (3.14): we solve a discrete problem in spaces $V_{h}, P_{h}$, with the first equation being the same as in (3.2), and the second one as in (3.14).
} 
Using (3.21) and (3.22) in (3.20) and dividing both sides by $\alpha_{c}\left\|\nabla \delta \mathbf{u}-\nabla \delta \widehat{\mathbf{u}}_{h}\right\|$, we eventually obtain

$$
\left\|\nabla \delta \mathbf{u}-\nabla \delta \widehat{\mathbf{u}}_{h}\right\| \leq c \frac{M}{\alpha_{c}} h^{2}\left\|\delta q_{\sigma}\right\|_{H^{3}(I)} .
$$

The second term in (3.19) can be estimated using the problems (3.14) and (3.18), fulfilled by $\delta \mathbf{u}_{h}, \delta \widehat{\mathbf{u}}_{h}$, together with the coercivity of $a$ and the continuity of the forms involved in such problems. We can thus obtain:

$$
\begin{aligned}
& \alpha_{c}\left\|\nabla \delta \widehat{\mathbf{u}}_{h}-\nabla \delta \mathbf{u}_{h}\right\|^{2} \leq a\left(q_{\sigma}\right)\left(\delta \widehat{\mathbf{u}}_{h}-\delta \mathbf{u}_{h}, \delta \widehat{\mathbf{u}}_{h}-\delta \mathbf{u}_{h}\right) \\
& =-\dot{a}\left(q_{\sigma}, \delta q\right)\left(\mathbf{u}-\mathbf{u}_{h}, \delta \widehat{\mathbf{u}}_{h}-\delta \mathbf{u}_{h}\right)-\dot{b}\left(q_{\sigma}, \delta q\right)\left(\delta \widehat{\mathbf{u}}_{h}-\delta \mathbf{u}_{h}, p-p_{h}\right) \\
& \quad-b\left(q_{\sigma}\right)\left(\delta \widehat{\mathbf{u}}_{h}-\delta \mathbf{u}_{h}, \delta \widehat{p}_{h}-\delta p_{h}\right) \\
& \leq c\|\delta q\|_{H^{2}(I)}\left(\left\|\nabla \mathbf{u}-\nabla \mathbf{u}_{h}\right\|+\left\|p-p_{h}\right\|\right)\left\|\nabla \delta \widehat{\mathbf{u}}_{h}-\nabla \delta \mathbf{u}_{h}\right\|,
\end{aligned}
$$

where the last inequality holds because $b\left(q_{\sigma}\right)\left(\delta \widehat{\mathbf{u}}_{h}, \pi_{h}\right)=b\left(q_{\sigma}\right)\left(\delta \mathbf{u}_{h}, \pi_{h}\right) \quad \forall \pi_{h} \in P_{h}$. After dividing by $\| \nabla \delta \widehat{\mathbf{u}}_{h}-$ $\nabla \delta \mathbf{u}_{h} \|$ both sides of (3.23), the right-hand side can be controlled as in the first point of the present lemma, leading to

$$
\left\|\nabla \delta \widehat{\mathbf{u}}_{h}-\nabla \delta \mathbf{u}_{h}\right\| \leq c h^{2}\left\|\delta q_{\sigma}\right\|_{H^{3}(I)} .
$$

Now we have to deal with pressure error terms in (3.19): taking a generic $\pi_{h} \in P_{h}$, the first term can be split as follows:

$$
\left\|\delta p-\delta \widehat{p}_{h}\right\| \leq\left\|\delta p-\pi_{h}\right\|+\left\|\pi_{h}-\delta \widehat{p}_{h}\right\| .
$$

We remark that, since inequality (3.25) holds for any $\pi_{h} \in P_{h}$, it holds also taking the infimum w.r.t. $\pi_{h}$. The infimum of the first term is directly controlled by $c h^{2}\left\|\delta q_{\sigma}\right\|_{H^{3}(I)}$ thanks to the interpolation error estimate (3.17) and the boundedness of $\|\delta p\|_{H^{2}\left(\Omega_{0}\right)}$ asserted in Assumption 3.16. The second term goes to zero when passing to the infimum, since $\delta \widehat{p}_{h} \in P_{h}$.

Finally, for the last term in (3.19) we exploit LBB condition (3.11) and proceed as follows:

$$
\begin{aligned}
\left\|\delta \widehat{p}_{h}-\delta p_{h}\right\| & \leq \sup _{\mathbf{v}_{h} \in V_{h}} \frac{b\left(q_{\sigma}\right)\left(\mathbf{v}_{h}, \delta \widehat{p}_{h}-\delta p_{h}\right)}{\widehat{\beta}\left\|\nabla \mathbf{v}_{h}\right\|} \\
& =\sup _{\mathbf{v}_{h} \in V_{h}} \frac{-\dot{a}\left(q_{\sigma}, \delta q_{\sigma}\right)\left(\mathbf{u}-\mathbf{u}_{h}, \mathbf{v}_{h}\right)-\dot{b}\left(q_{\sigma}, \delta q_{\sigma}\right)\left(\mathbf{v}_{h}, p-p_{h}\right)-a\left(q_{\sigma}\right)\left(\delta \widehat{\mathbf{u}}_{h}-\delta \mathbf{u}_{h}, \mathbf{v}_{h}\right)}{\widehat{\beta}\left\|\nabla \mathbf{v}_{h}\right\|} \\
& \leq \frac{1}{\widehat{\beta}}\left[c\left\|\delta q_{\sigma}\right\|_{H^{3}(I)}\left(\left\|\nabla \mathbf{u}-\nabla \mathbf{u}_{h}\right\|+\left\|p-p_{h}\right\|\right)+M\left\|\nabla \delta \widehat{\mathbf{u}}_{h}-\nabla \delta \mathbf{u}_{h}\right\|\right] .
\end{aligned}
$$

From estimate (3.24) and point (a) of the present lemma, we get the desired bound $c h^{2}\left\|\delta q_{\sigma}\right\|_{H^{3}(I)}$. Collecting the estimates for the four terms in (3.19) yields the validity of point (b).

Finally, we prove (c), employing the regularity result for $S^{\prime \prime}\left(q_{\sigma}\right)\left(\delta q_{\sigma}, \delta q_{\sigma}\right)$ given at the third point of Assumption 3.16. The only difference from the previous point is the "intermediate derivative" $\left(\delta \delta \widehat{\mathbf{u}}_{h}, \delta \delta \widehat{p}_{h}\right) \in V_{h} \times P_{h}$, defined as the solution of the following problem:

$$
\left\{\begin{array}{rlrl}
a\left(q_{\sigma}\right) & \left(\delta \delta \widehat{\mathbf{u}}_{h}, \mathbf{v}_{h}\right)+b\left(q_{\sigma}\right)\left(\mathbf{v}_{h}, \delta \delta \widehat{p}_{h}\right)= & \\
\ddot{F}\left(q_{\sigma}, \delta q_{\sigma}, \delta q_{\sigma}\right)\left(\mathbf{v}_{h}\right)-\ddot{a}\left(q_{\sigma}, \delta q_{\sigma}, \delta q_{\sigma}\right)\left(\mathbf{u}, \mathbf{v}_{h}\right)-\ddot{b}\left(q_{\sigma}, \delta q_{\sigma}, \delta q_{\sigma}\right)\left(\mathbf{v}_{h}, p\right) & & \forall \mathbf{v}_{h} \in V_{h}, \\
& -2 \dot{a}\left(q_{\sigma}, \delta q_{\sigma}\right)\left(\delta \mathbf{u}, \mathbf{v}_{h}\right)-2 \dot{b}\left(q_{\sigma}, \delta q_{\sigma}\right)\left(\mathbf{v}_{h}, \delta p\right) & \\
b\left(q_{\sigma}\right)\left(\delta \delta \widehat{\mathbf{u}}_{h}, \pi_{h}\right)=\ddot{G}\left(q_{\sigma}, \delta q_{\sigma}, \delta q_{\sigma}\right)\left(\pi_{h}\right)-\ddot{b}\left(q_{\sigma}, \delta q_{\sigma}, \delta q_{\sigma}\right)\left(\mathbf{u}_{h}, \pi_{h}\right) & & \forall \pi_{h} \in P_{h} . \\
-2 \dot{b}\left(q_{\sigma}, \delta q_{\sigma}\right)\left(\delta \mathbf{u}_{h}, \pi_{h}\right) &
\end{array}\right.
$$

All the previous steps performed to estimate $S^{\prime}-S_{h}^{\prime}$ can be easily adapted to the present context.

A direct consequence of the previous lemma is the following convergence result for the discrete functional. 
Lemma 3.20. $\forall q_{\sigma} \in Q_{\sigma}^{a d}, \delta q_{\sigma} \in \delta Q_{\sigma}$ it holds

(a) $\left|j\left(q_{\sigma}\right)-j_{h}\left(q_{\sigma}\right)\right| \leq c h^{2}$

(b) $\left|j^{\prime}\left(q_{\sigma}\right)\left(\delta q_{\sigma}\right)-j_{h}^{\prime}\left(q_{\sigma}\right)\left(\delta q_{\sigma}\right)\right| \leq c h^{2}\left\|\delta q_{\sigma}\right\|_{H^{3}(I)}$;

(c) $\left|j^{\prime \prime}\left(q_{\sigma}\right)\left(\delta q_{\sigma}, \delta q_{\sigma}\right)-j_{h}^{\prime \prime}\left(q_{\sigma}\right)\left(\delta q_{\sigma}, \delta q_{\sigma}\right)\right| \leq c h^{2}\left\|\delta q_{\sigma}\right\|_{H^{3}(I)}^{2}$.

Proof.

Let us fix a $q_{\sigma} \in Q_{\sigma}^{a d}, \delta q_{\sigma} \in \delta Q_{\sigma}$ and define $(\mathbf{u}, p)=S\left(q_{\sigma}\right),(\delta \mathbf{u}, \delta p)=S^{\prime}\left(q_{\sigma}\right)\left(\delta q_{\sigma}\right),(\delta \delta \mathbf{u}, \delta \delta p)=$ $S^{\prime \prime}\left(q_{\sigma}\right)\left(\delta q_{\sigma}, \delta q_{\sigma}\right)$.

Let us first prove (a). It it easy to show that the following holds

$$
\begin{gathered}
\left|j\left(q_{\sigma}\right)-j_{h}\left(q_{\sigma}\right)\right|=\left|\left(\left(\nabla \mathbf{u}-\nabla \mathbf{u}_{h}\right) A_{q_{\sigma}}, \nabla \mathbf{u}+\nabla \mathbf{u}_{h}\right)\right| \\
\leq\left\|A_{q_{\sigma}}\right\|_{\infty}\left(\|\nabla \mathbf{u}\|+\left\|\nabla \mathbf{u}_{h}\right\|\right)\left\|\nabla \mathbf{u}-\nabla \mathbf{u}_{h}\right\| \leq c h^{2},
\end{gathered}
$$

where the last inequality employs the boundedness of $A_{q_{\sigma}}, \nabla \mathbf{u}, \nabla \mathbf{u}_{h}$ and Lemma 3.19.

Now we prove (b), according to the following steps:

$$
\begin{aligned}
& \left|j^{\prime}\left(q_{\sigma}\right)\left(\delta q_{\sigma}\right)-j_{h}^{\prime}\left(q_{\sigma}\right)\left(\delta q_{\sigma}\right)\right| \leq\left|\left(\left(\nabla \mathbf{u}-\nabla \mathbf{u}_{h}\right) A_{q_{\sigma}, \delta q_{\sigma}}^{\prime}, \nabla \mathbf{u}+\nabla \mathbf{u}_{h}\right)\right| \\
& +2\left|\left(\left(\nabla \delta \mathbf{u}-\nabla \delta \mathbf{u}_{h}\right) A_{q_{\sigma}}, \nabla \mathbf{u}\right)\right|+2\left|\left(\nabla \delta \mathbf{u}_{h} A_{q_{\sigma}}, \nabla \mathbf{u}-\nabla \mathbf{u}_{h}\right)\right| \\
& \leq c h^{2}\left\|\delta q_{\sigma}\right\|_{H^{3}(I)} .
\end{aligned}
$$

Indeed, it holds $\left\|A_{q_{\sigma}, \delta q_{\sigma}}^{\prime}\right\|_{\infty} \leq c\left\|\delta q_{\sigma}\right\|_{H^{2}(I)}$ (see Prop. 3.4) while $\|\nabla \mathbf{u}\|$ and $\left\|\nabla \delta \mathbf{u}_{h}\right\|$ are controlled thanks to the continuous and discrete versions of Theorem 3.5, and the discretization error terms are bounded through Lemma 3.19.

Finally, we prove (c), as follows:

$$
\begin{aligned}
& \left|j^{\prime \prime}\left(q_{\sigma}\right)\left(\delta q_{\sigma}, \delta q_{\sigma}\right)-j_{h}^{\prime \prime}\left(q_{\sigma}\right)\left(\delta q_{\sigma}, \delta q_{\sigma}\right)\right| \leq\left|\left(\left(\nabla \mathbf{u}-\nabla \mathbf{u}_{h}\right) A_{q_{\sigma}, \delta q_{\sigma}, \delta q_{\sigma}}^{\prime \prime}, \nabla \mathbf{u}+\nabla \mathbf{u}_{h}\right)\right| \\
& \quad+4\left|\left(\left(\nabla \delta \mathbf{u}-\nabla \delta \mathbf{u}_{h}\right) A_{q_{\sigma}, \delta q_{\sigma}}^{\prime}, \nabla \mathbf{u}\right)\right|+4\left|\left(\nabla \delta \mathbf{u}_{h} A_{q_{\sigma}, \delta q_{\sigma}}^{\prime}, \nabla \mathbf{u}-\nabla \mathbf{u}_{h}\right)\right| \\
& \quad+2\left|\left(\left(\nabla \delta \mathbf{u}-\nabla \delta \mathbf{u}_{h}\right) A_{q_{\sigma}}, \nabla \delta \mathbf{u}+\nabla \delta \mathbf{u}_{h}\right)\right| \\
& \quad+2\left|\left(\left(\nabla \delta \delta \mathbf{u}-\nabla \delta \delta \mathbf{u}_{h}\right) A_{q_{\sigma}}, \nabla \mathbf{u}\right)\right|+2\left|\left(\nabla \delta \delta \mathbf{u}_{h} A_{q_{\sigma}}, \nabla \mathbf{u}-\nabla \mathbf{u}_{h}\right)\right| .
\end{aligned}
$$

To bound the terms not involving $\delta \delta \mathbf{u}$ and $\delta \delta \mathbf{u}_{h}$, one can employ Proposition 3.4 to handle the matrix terms, together with similar techniques already used to prove (a) and (b). To bound the last two terms, we have to apply Lemma 3.19 , point $\mathrm{c}$, and the discrete version of Theorem 3.5 in order to provide estimates for $\left\|\nabla \delta \delta \mathbf{u}-\nabla \delta \delta \mathbf{u}_{h}\right\|$ and $\left\|\nabla \delta \delta \mathbf{u}_{h}\right\|$.

Finally, collecting the previous results, we can prove the main result of this section, under the assumption that the optimal control $\bar{q}$ is in $H^{5}(I)$.

Theorem 3.21 (A priori convergence estimates). Let Assumptions 3.9, 3.10 and 3.16 hold. If a local solution $\bar{q}$ of $(2.9)$ belongs to $H^{5}(I)$, then there exists a sequence $\left\{\bar{q}_{\sigma, h}\right\}_{\sigma, h>0}$ of local optimal solutions of the discrete problem

$$
\min _{q_{\sigma} \in Q_{\sigma}^{a d}} j_{h}\left(q_{\sigma}\right)
$$

such that

$$
\begin{aligned}
\left\|\bar{q}-\bar{q}_{\sigma, h}\right\|_{H^{3}(I)} & =\mathcal{O}\left(\sigma^{2}+h^{2}\right), \\
\left\|S(\bar{q})-S_{h}\left(\bar{q}_{\sigma, h}\right)\right\|_{V \times P} & =\mathcal{O}\left(\sigma^{2}+h^{2}\right), \\
\left|j(\bar{q})-j_{h}\left(\bar{q}_{\sigma, h}\right)\right| & =\mathcal{O}\left(\sigma^{2}+h^{2}\right) .
\end{aligned}
$$


Proof. Let $\bar{q}_{\sigma}, \bar{q}_{\sigma, h}$ denote the optimal controls for the semi-discrete problem (3.5) and the fully discretized problem (3.26), respectively. The Mean Value Theorem ensures the existence of $t \in(0,1)$ such that, with $\xi=t \bar{q}_{\sigma}+(1-t) \bar{q}_{\sigma, h}$, we have

$$
j_{h}^{\prime}\left(\bar{q}_{\sigma}\right)\left(\delta q_{\sigma}\right)-j_{h}^{\prime}\left(\bar{q}_{\sigma, h}\right)\left(\delta q_{\sigma}\right)=j_{h}^{\prime \prime}(\xi)\left(\delta q_{\sigma}, \bar{q}_{\sigma}-\bar{q}_{\sigma, h}\right) \quad \forall \delta q_{\sigma} \in \delta Q_{\sigma} .
$$

Applying Lemma 3.14 and taking $\bar{q}_{\sigma}-\bar{q}_{\sigma, h}$ as a variation, we get:

$$
\begin{aligned}
\frac{\delta_{2}}{2}\left\|\bar{q}_{\sigma}-\bar{q}_{\sigma, h}\right\|_{H^{3}(I)}^{2} \leq & j^{\prime \prime}(\xi)\left(\bar{q}_{\sigma}-\bar{q}_{\sigma, h}, \bar{q}_{\sigma}-\bar{q}_{\sigma, h}\right) \\
\leq & j_{h}^{\prime \prime}(\xi)\left(\bar{q}_{\sigma}-\bar{q}_{\sigma, h}, \bar{q}_{\sigma}-\bar{q}_{\sigma, h}\right) \\
& +\left|j^{\prime \prime}(\xi)\left(\bar{q}_{\sigma}-\bar{q}_{\sigma, h}, \bar{q}_{\sigma}-\bar{q}_{\sigma, h}\right)-j_{h}^{\prime \prime}(\xi)\left(\bar{q}_{\sigma}-\bar{q}_{\sigma, h}, \bar{q}_{\sigma}-\bar{q}_{\sigma, h}\right)\right| \\
\leq & j_{h}^{\prime}\left(\bar{q}_{\sigma}\right)\left(\bar{q}_{\sigma}-\bar{q}_{\sigma, h}\right)-j_{h}^{\prime}\left(\bar{q}_{\sigma, h}\right)\left(\bar{q}_{\sigma}-\bar{q}_{\sigma, h}\right)+c_{1} h^{2}\left\|\bar{q}_{\sigma}-\bar{q}_{\sigma, h}\right\|_{H^{3}(I)}^{2},
\end{aligned}
$$

where the last inequality is obtained by (3.27) and Lemma 3.20(c). Using the fact that $j_{h}^{\prime}\left(\bar{q}_{\sigma, h}\right)\left(\bar{q}_{\sigma}-\bar{q}_{\sigma, h}\right)=j^{\prime}\left(\bar{q}_{\sigma}\right)\left(\bar{q}_{\sigma}-\bar{q}_{\sigma, h}\right)=0$ in the right-hand side of (3.3), and then applying Lemma 3.20(b), we obtain:

$$
\begin{aligned}
\frac{\delta_{2}}{2}\left\|\bar{q}_{\sigma}-\bar{q}_{\sigma, h}\right\|_{H^{2}(I)}^{2} & \leq j_{h}^{\prime}\left(\bar{q}_{\sigma}\right)\left(\bar{q}_{\sigma}-\bar{q}_{\sigma, h}\right)-j^{\prime}\left(\bar{q}_{\sigma}\right)\left(\bar{q}_{\sigma}-\bar{q}_{\sigma, h}\right)+c_{1} h^{2}\left\|\bar{q}_{\sigma}-\bar{q}_{\sigma, h}\right\|_{H^{3}(I)}^{2} \\
& \leq c_{2} h^{2}\left\|\bar{q}_{\sigma}-\bar{q}_{\sigma, h}\right\|_{H^{3}(I)}+c_{1} h^{2}\left\|\bar{q}_{\sigma}-\bar{q}_{\sigma, h}\right\|_{H^{3}(I)}^{2} .
\end{aligned}
$$

Therefore, for sufficiently small $h$, i.e. for

$$
h \leq\left(\frac{\delta_{2}}{2 c_{1}}\right)^{1 / 2}
$$

and employing Proposition 3.6, the following convergence error estimate holds:

$$
\left\|\bar{q}-\bar{q}_{\sigma, h}\right\|_{H^{3}(I)} \leq\left\|\bar{q}-\bar{q}_{\sigma}\right\|_{H^{3}(I)}+\left\|\bar{q}_{\sigma}-\bar{q}_{\sigma, h}\right\|_{H^{3}(I)}=\mathcal{O}\left(\sigma^{2}+h^{2}\right) .
$$

This result yields the second point of the thesis, since

$$
\left\|S(\bar{q})-S_{h}\left(\bar{q}_{\sigma, h}\right)\right\|_{V \times P} \leq\left\|S(\bar{q})-S\left(\bar{q}_{\sigma, h}\right)\right\|_{V \times P}+\left\|S\left(\bar{q}_{\sigma, h}\right)-S_{h}\left(\bar{q}_{\sigma, h}\right)\right\|_{V \times P},
$$

and the desired estimate for $S_{h}$ follows from applying Lemmas 3.12 and 3.19 to the two terms at right-hand side of (3.28). An analogous argument, using Lemmas 3.13 and 3.20, yields the estimate for $j_{h}$.

\section{Optimality COnditions}

In this section, we want to write the first order optimality condition

$$
j^{\prime}(\bar{q})(\delta q)=0 \quad \forall \delta q \in \delta Q,
$$

in terms of the reference-domain state solution $S(q)$. This is instrumental to design the descent algorithm (see Sect. 5) for the numerical approximation of the minimization problem (2.9). Since the expression of the gradient is exploited only in the numerical tests, we proceed formally in its derivation, as generally accepted and done in the field of numerical shape optimization (see, e.g., $[1,11,22,30]$ ).

In order to simplify computation, instead of differentiating the expression for functional $j$ given in (2.9), we exploit the fact that $j(q)=\widetilde{j}(q)$ for any $q \in Q^{a d}$, and start from the expression of $\widetilde{j}$ defined in (2.7), i.e.,

$$
\widetilde{j}(q)=\int_{\Omega_{q}}|\nabla \widetilde{\mathbf{u}}|^{2} \mathrm{~d} \Omega+\frac{\alpha}{2}\left\|q^{\prime \prime}\right\|_{L^{2}(I)}^{2}+\frac{\beta}{2}\left(\int_{I} q(x) \mathrm{d} x-\bar{V}\right)^{2},
$$


where $\widetilde{\mathbf{u}}: \Omega_{q} \rightarrow \mathbb{R}^{2}$, together with $\widetilde{p}: \Omega_{q} \rightarrow \mathbb{R}$, is the solution of Stokes problem (2.1). Through the Hadamard formula (see, e.g., [35]), we are going to write the derivative $\widetilde{j^{\prime}}(q)(\delta q)$ in terms of $\widetilde{\mathbf{u}}$ and the solution of an adjoint problem, and then to pull back the resulting expression into the reference domain.

The so-called shape-derivative of $(\widetilde{\mathbf{u}}, \widetilde{p})$ can be defined as the solution $(\widetilde{\delta \mathbf{u}}, \widetilde{\delta p})$ of the following problem (see, e.g., [30]):

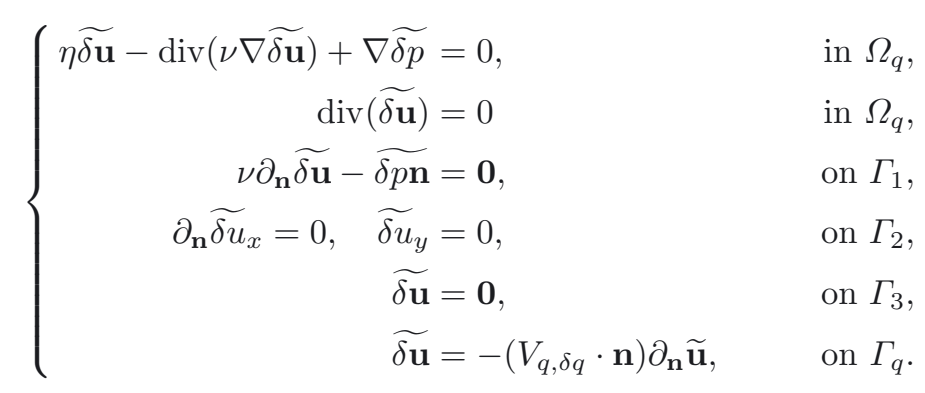

where $V_{q, \delta q}$ is the vector field describing a transformation from $\Omega_{q}$ to $\Omega_{q+\delta q}$, given by

$$
V_{q, \delta q}(x, y)=\left(\begin{array}{c}
0 \\
\frac{1-y}{1-q(x)} \delta q(x)
\end{array}\right)
$$

Differentiating expression (4.2) along direction $\delta q$, one obtains

$$
\widetilde{j}^{\prime}(q)(\delta q)=2(\nabla \widetilde{\mathbf{u}}, \nabla \widetilde{\delta \mathbf{u}})_{\Omega_{q}}+\int_{\Gamma_{q}}|\nabla \widetilde{\mathbf{u}}|^{2} V_{q, \delta q} \cdot \mathbf{n} \mathrm{d} \Gamma+\alpha\left(q^{\prime \prime}, \delta q^{\prime \prime}\right)_{I}+\beta\left(\int_{I} q(x) \mathrm{d} x-\bar{V}\right) \int_{I} \delta q(x) \mathrm{d} x .
$$

In order to make the dependence of $\widetilde{j}^{\prime}(q)(\delta q)$ on $\delta q$ completely explicit, we introduce the adjoint state $(\widetilde{\mathbf{z}}, \widetilde{s})$, solution of the following adjoint problem:

$$
\left\{\begin{aligned}
\eta \widetilde{\mathbf{z}}-\operatorname{div}(\nu \nabla \widetilde{\mathbf{z}})+\nabla \widetilde{s} & =-2 \Delta \widetilde{\mathbf{u}}, & & \text { in } \Omega_{q}, \\
\operatorname{div} \widetilde{\mathbf{z}} & =0, & & \text { in } \Omega_{q}, \\
\nu \partial_{\mathbf{n}} \widetilde{\mathbf{z}}-\widetilde{s} \mathbf{n} & =2 \partial_{\mathbf{n}} \widetilde{\mathbf{u}}, & & \text { on } \Gamma_{1}, \\
\nu \partial_{\mathbf{n}} \widetilde{z}_{x}=2 \partial_{\mathbf{n}} \widetilde{u}_{x}, & \widetilde{z}_{y}=0, & & \text { on } \Gamma_{2}, \\
\widetilde{\mathbf{z}} & =\mathbf{0}, & & \text { on } \Gamma_{q} \cup \Gamma_{3} .
\end{aligned}\right.
$$

Using both problems (4.3) and (4.4), and exploiting integration by parts, we can re-write the derivative of the functional as depending only on $\widetilde{\mathbf{u}}, \widetilde{\mathbf{z}}, q, \delta q$, and no more on $\widetilde{\delta \mathbf{u}}$, obtaining the following Hadamard formula for $\widetilde{j^{\prime}}$ :

$$
\widetilde{j}^{\prime}(q)(\delta q)=\int_{\Gamma_{q}} \partial_{\mathbf{n}} \widetilde{\mathbf{u}} \cdot\left(\nu \partial_{\mathbf{n}} \widetilde{\mathbf{z}}-\partial_{\mathbf{n}} \widetilde{\mathbf{u}}\right)\left(V_{q, \delta q} \cdot \mathbf{n}\right) \mathrm{d} \Gamma+\alpha\left(q^{\prime \prime}, \delta q^{\prime \prime}\right)_{I}+\beta\left(\int_{I} q(x) \mathrm{d} x-\bar{V}\right) \int_{I} \delta q(x) \mathrm{d} x .
$$

Since we adopt a reference domain approach, we pass from (4.5) to an expression depending on the pulled-back state variables $(\mathbf{u}, p)=S(q)$ and adjoint velocity $\mathbf{z}=\widetilde{\mathbf{z}} \circ T_{q}$ : employing changes of variables from $\Omega_{q}$ to $\Omega_{0}$, and from $\Gamma_{q}$ to $I$, the following result holds.

Lemma 4.1. Given the functional $j(q)$ defined as in (2.9), its Gateaux-derivative in $q$ along direction $\delta q$ is given by

$$
j^{\prime}(q)(\delta q)=\alpha\left(q^{\prime \prime}, \delta q^{\prime \prime}\right)_{I}+(\Psi(q), \delta q)_{I} \quad \forall q \in Q^{a d}, \delta q \in \delta Q
$$


where $\Psi(q): I \rightarrow \mathbb{R}$ is defined as

$$
\begin{aligned}
\Psi(q)(x) & =\Psi_{1}(q)(x)+\Psi_{2}(q)(x):=\beta\left(\int_{I} q(t) \mathrm{d} t-\bar{V}\right) \\
& +\frac{1}{1+q^{\prime}(x)^{2}}\left[\nabla \mathbf{u} A_{q} \mathbf{n}\right](x, 0) \cdot\left[(\nabla \mathbf{u}-\nu \nabla \mathbf{z}) A_{q} \mathbf{n}\right](x, 0),
\end{aligned}
$$

with $\mathbf{n}=(0,-1)$ denoting the outward normal versor of the lower boundary segment $\Gamma_{0}$ of the domain $\Omega_{0}$.

\section{Numerical RESUlts}

In this section, we present two sets of numerical results. The numerical implementation has been carried out basing on the FEniCS project (see [29] and http://fenicsproject.org), and the optimal solution is obtained iteratively, using the following gradient method [31]:

- Gradient method iteration

Given $q_{\text {old }}$ from the previous iteration, set the descent step length $\varepsilon$ to the initial value $\widehat{\varepsilon}>0$. Then,

(1) solve state and adjoint problems in order to obtain $(\mathbf{u}, p),(\mathbf{z}, s)$

(2) build $\nabla j\left(q_{\text {old }}\right)$

(3) project $\nabla j\left(q_{\text {old }}\right)$ on the set of admissible variations, obtaining $G$

(4) restrict $G$ on $\Gamma_{0}$ and then map it to $I$, to get $g$

(5) back-tracking: set $q_{\text {new }}=q_{\text {old }}-\varepsilon g$

while $j\left(q_{\text {new }}\right)>j\left(q_{\text {old }}\right)$ and $\varepsilon>\varepsilon_{\min }$ do:

(a) update: $q_{\text {new }}=q_{\text {old }}-\varepsilon g$

(b) $\varepsilon=\varepsilon / 2$

In general, the functional gradient $\nabla j\left(q_{\text {old }}\right)$, obtained as in Lemma 4.1, is not an admissible variation, since one cannot prove the existence of some $\varepsilon>0$ such that $q=q_{\text {old }}-\varepsilon \nabla j\left(q_{\text {old }}\right)$ satisfies

$$
q(0)=q(1)=0 .
$$

This is why in the above gradient method the projection step (3) is required. The gradient $\nabla j\left(q_{o l d}\right)$ is projected onto $H_{\partial \Omega_{0} \backslash \Gamma_{0}}^{1}\left(\Omega_{0}\right)$ solving the following problem:

$$
\begin{cases}-\Delta G+G=0, & \text { in } \Omega_{0}, \\ G=0, & \text { on } \partial \Omega_{0} \backslash \Gamma_{0}, \\ -\partial_{\mathbf{n}} G=-\nabla j\left(q_{\text {old }}\right), & \text { on } \Gamma_{0} .\end{cases}
$$

Then, step (4) of the algorithm reduces $G$, defined on $\Omega_{0}$, to a function $g$ belonging to the space of controls.

The results obtained by the application of the above algorithm to the shape optimization problem (2.9) are now presented and discussed. Two different functionals will be considered in the following two test cases.

Remark 5.1. We remark that we use finite element discretization, with $\mathbb{P}_{2}-\mathbb{P}_{1}$ pair for state velocity and pressure and with piecewise linear basis functions for the control. As we will see, even if the polynomial degree for controls is not as high as assumed in the derivation of a priori estimates, the numerical results comply the theoretical ones. In these numerical tests, we consider a unique discretization parameter, i.e. we set $\sigma=h$. 


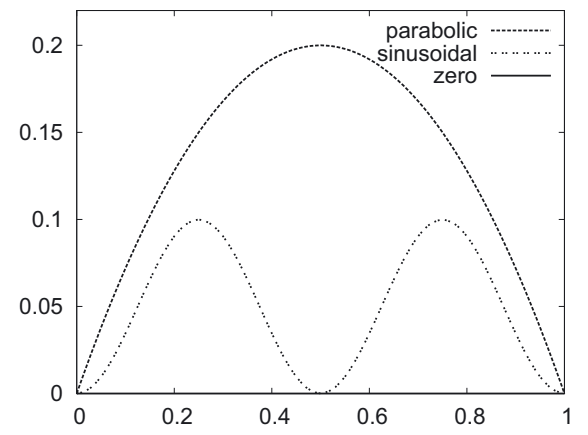

(a) Initial configurations

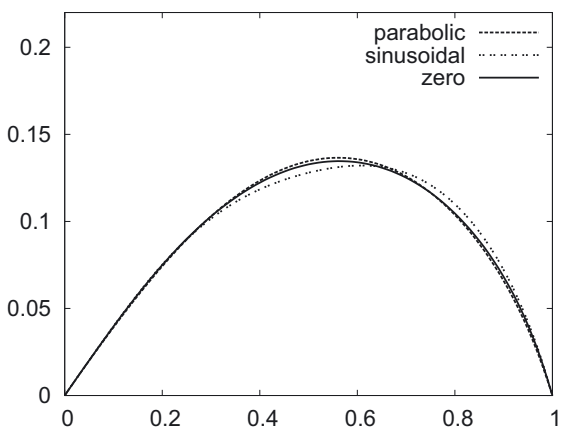

(b) Final configurations

FIgURE 2. Independence of the optimal control from the initial configuration, for $\alpha=10$, $\beta=10000, \bar{V}=[0.7$ times the initial area of the parabolic case $]$.

\subsection{Test case 1}

In this first test case, we take into account the following functional:

$$
\widetilde{j}(q)=\int_{\Omega_{0}}|\nabla \mathbf{u}|^{2} \mathrm{~d} \Omega+\frac{\alpha}{2} \int_{\Gamma_{q}} \mathrm{~d} \Gamma+\frac{\beta}{2}\left(\int_{I} q(x) \mathrm{d} x-\bar{V}\right)^{2} .
$$

Its counterpart on the pulled-back formulation (2.8) reads

$$
j(q)=\int_{\Omega_{0}} \gamma_{q}\left|\nabla \mathbf{u} D T_{q}^{-1}\right|^{2} \mathrm{~d} \Omega+\frac{\alpha}{2} \int_{I} \sqrt{1+\left(q^{\prime}(x)\right)^{2}} \mathrm{~d} x+\frac{\beta}{2}\left(\int_{I} q(x) \mathrm{d} x-\bar{V}\right)^{2} .
$$

The gradient of this functional is given by

$$
\begin{aligned}
\nabla j(q)= & \left.\left.\frac{1}{1+\left(q^{\prime}\right)^{2}}\left[\nabla \mathbf{u} A_{q} \mathbf{n}\right]\right|_{\Gamma_{0}} \cdot\left[(\nabla \mathbf{u}-\nu \nabla \mathbf{z}) A_{q} \mathbf{n}\right]\right|_{\Gamma_{0}} \\
& -\frac{\alpha}{2} \frac{q^{\prime \prime}}{\left(1+\left(q^{\prime}\right)^{2}\right)^{3 / 2}}+\beta\left(\int_{I} q(x) \mathrm{d} x-\bar{V}\right) .
\end{aligned}
$$

The regularization term considered in (5.1) is often used in the literature (see, e.g., [13,30]) and it consists in the penalization of the perimeter of the moving portion $\Gamma_{q}$ of the domain boundary. This new term is simpler to handle than the curvature term $\left\|q^{\prime \prime}\right\|_{L^{2}(I)}^{2}$ : indeed, using the original term would require the introduction of a further adjoint problem, to extract the Riesz representative in $L^{2}(I)$ of the functional $\delta q \mapsto\left(q^{\prime \prime}, \delta q^{\prime \prime}\right)_{I}$. Moreover, the perimeter term can be supposed to generally act in the same way as the curvature term, since a shorter perimeter corresponds to less oscillations, and vice versa.

We first analyze the dependence of the optimal solution on the initial configuration. We consider three different initial solutions, defined by a parabolic function $\left(q(x)=0.2\left[1-4(x-0.5)^{2}\right]\right)$, a sinusoidal function $\left(q(x)=0.1 \sin (2 \pi x)^{2}\right)$, and the flat function $(q(x) \equiv 0)$. As shown in Figure 2, starting from different initial controls, very similar optimal controls are obtained. The final configurations in Figure $2 \mathrm{~b}$ are reached in less than 10 iterations, with $\widehat{\varepsilon}=0.1, \varepsilon_{\min }=10^{-8}$, and the reaching of $\varepsilon \leq \varepsilon_{\min }$ as the stop criterion on the iterations of the gradient method.

In Figure 3 the dependence of the solution on the value of the penalty parameters has also been analyzed, starting from the parabolic configuration. With respect to the choice of the parameter $\alpha$, a minimum value has to be exceeded in order to prevent the gradient method from converging to a local, sub-optimal minimum. 


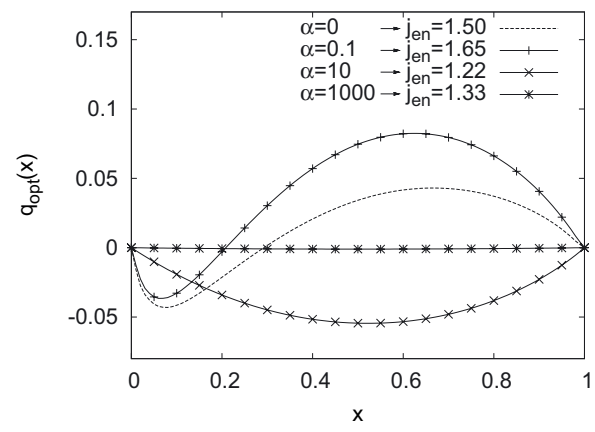

(a) Varying $\alpha ; \beta=0$

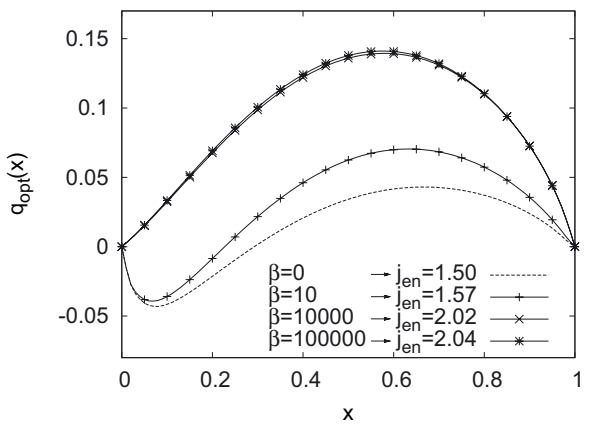

(b) Varying $\beta ; \alpha=0$

FiguRE 3. Final controls obtained by the optimization algorithm for different values of the penalty parameters $\left(j_{\text {en }}\right.$ is the energetic term of the functional $j$ ).

Indeed, Figure 3a shows that for lower values of $\alpha$, oscillating controls are found at the end of the optimization algorithm, though the value of the energetic part of the functional in such configurations is higher than the ones corresponding to $\alpha=10,1000$. Moreover, a maximum value must not be exceeded, otherwise the regularization parameter dominates too much in the total functional value, leading to a nearly flat optimal control. On the other hand, the parameter $\beta$ is only needed to be chosen greater than a minimum threshold, this in order to sufficiently enforce the volume constraint. Bearing in mind the above considerations, Figure 3 shows that the values $\alpha=10$ and $\beta=10000$ are possible effective values for the two penalty parameters.

\subsection{Test case 2}

In this section, we report a numerical convergence analysis, carried out to validate the a priori error estimates proved in Theorem 3.21. For this purpose, we would like to have an exact solution as a reference point. To this end, we take into account the following functional:

$$
\widetilde{j}(q)=\int_{\Omega_{q}}\left|\nabla \widetilde{\mathbf{u}}-\nabla \widetilde{\mathbf{u}}_{d}\right|^{2} \mathrm{~d} \Omega+\frac{\alpha}{2} \int_{\Gamma_{q}} \mathrm{~d} \Gamma
$$

with its pulled-back counterpart given by

$$
j(q)=\int_{\Omega_{0}}\left(\nabla \mathbf{u}-\nabla \mathbf{u}_{d}\right) A_{q}\left(\nabla \mathbf{u}-\nabla \mathbf{u}_{d}\right) \mathrm{d} \Omega+\frac{\alpha}{2} \int_{I} \sqrt{1+\left(q^{\prime}(x)\right)^{2}} \mathrm{~d} x .
$$

The velocity $\widetilde{\mathbf{u}}_{d}$ is obtained solving the Stokes problem (2.1) on a domain $\Omega_{q_{d}}$, identified by the given control function

$$
q_{d}=0.1+0.1 \cos (2 \pi(x-0.5)),
$$

and $\mathbf{u}_{d}=\widetilde{\mathbf{u}}_{d} \circ T_{q_{d}}$.

Indeed, if no penalty terms are active, the minimum for this functional is zero, and it is reached for $q=q_{d}$. The functional (5.2) is a slight generalization of the functional defined in (2.9), and the theoretical results presented in the previous sections can be easily generalized to the new functional.

Following the steps of Section 4, we can derive an expression for the shape gradient in $q$ :

$$
\nabla j(q)=-\frac{\alpha}{2} \frac{q^{\prime \prime}}{\left(1+\left(q^{\prime}\right)^{2}\right)^{3 / 2}}+\left.\left.\frac{1}{1+\left(q^{\prime}\right)^{2}}\left[\left(\nabla \mathbf{u}-\nabla \mathbf{u}_{d}\right) A_{q} \mathbf{n}\right]\right|_{\Gamma_{0}} \cdot\left[\left(\nabla \mathbf{u}-\nabla \mathbf{u}_{d}-\nu \nabla \mathbf{z}_{\mathbf{u}_{d}}\right) A_{q} \mathbf{n}\right]\right|_{\Gamma_{0}},
$$

where $\mathbf{z}_{\mathbf{u}_{d}}$ is the adjoint velocity variable, solution of a problem obtained from a minimal modification of (4.4), replacing any occurrence of $\widetilde{\mathbf{u}}$ with $\widetilde{\mathbf{u}}-\widetilde{\mathbf{u}}_{d}$. 


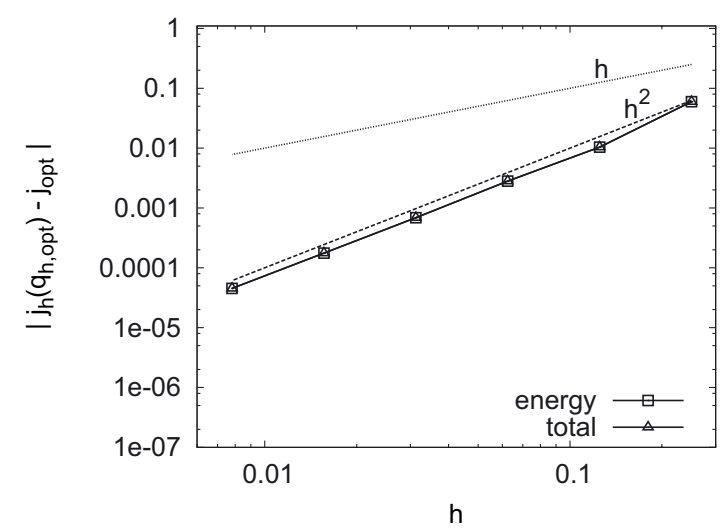

(a) $\alpha=0$

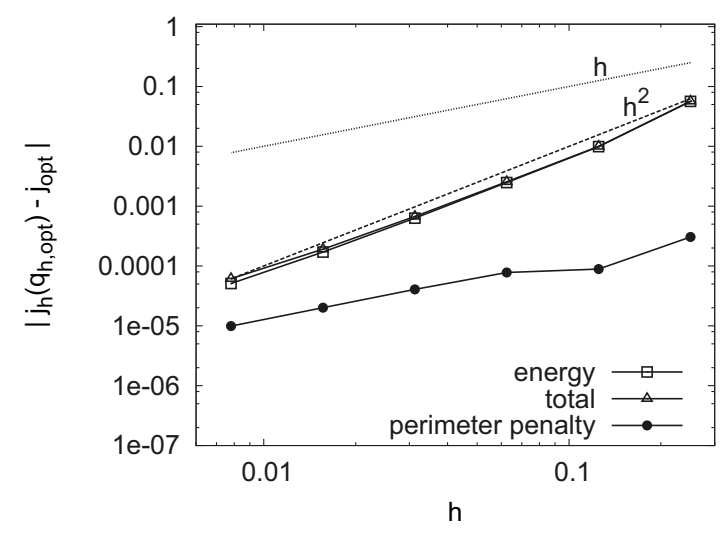

(c) $\alpha=0.1$

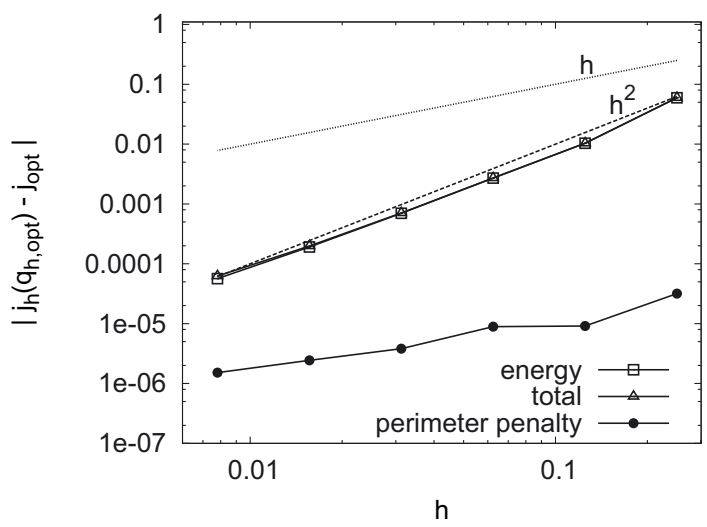

(b) $\alpha=0.01$

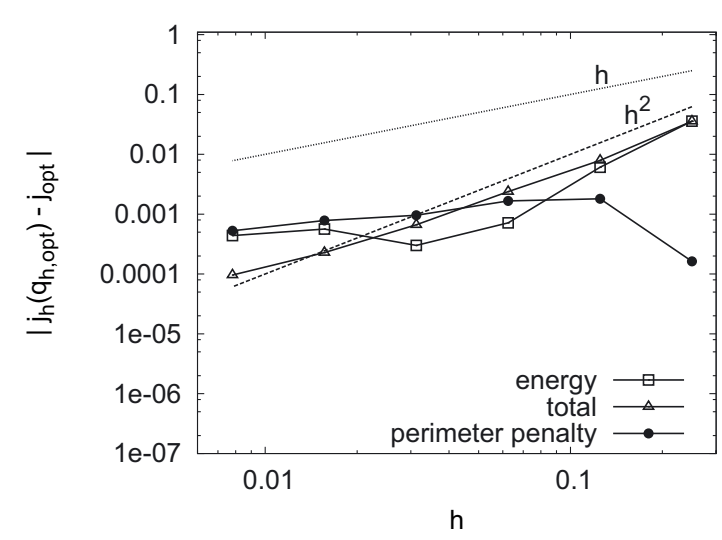

(d) $\alpha=1$

FiguRE 4. Spatial convergence of discrete functional value $j_{h}\left(q_{h, o p t}\right)$ to its reference value $j\left(q_{\text {opt }}\right)$. Each term of the functional is presented w.r.t. its corresponding term in $j\left(q_{\text {opt }}\right)$, which is known for $\alpha=0$, and obtained by Richardson extrapolation for $\alpha \neq 0$.

Based on the functional defined in (5.2), different spatial convergence tests have been carried out, taking four specific values for perimeter penalty parameter $\alpha$, namely $\alpha=0,0.01,0.1,1$.

The results reported in Figure 4 are in agreement with the a priori estimates of the convergence error proved in Theorem 3.21, since an approximately quadratic convergence order is obtained, for a broad spectrum of values of $h$. However, for $h \rightarrow 0$, the graphs in Figure 4 show a sort of saturation bending. A reason for this can be found in the stopping criterion of the optimization algorithm and in the lower bound imposed on the descent step length, that introduce a finite error. This influence is amplified as $\alpha$ grows, to the point of polluting the convergence behaviour, hence we do not report results for $\alpha>1$.

\section{Conclusions}

In this paper, we have studied a shape optimization problem, namely the minimization of the total energy dissipation for the low-Reynolds flow of a viscous, incompressible fluid, modeled by two-dimensional, steady Stokes equations. After the definition of the problem and the admissible set of control functions, we have reformulated the problem onto a reference domain, by means of a control-dependent map. The well-posedness of 
the transformed problem has been inspected, and particular attention has been devoted to the inf-sup condition on the form $b(q)$, obtaining a control-independent lower bound on the inf-sup constant. The existence of an optimal solution has also been proved, for the minimization problem at hand, and corresponding first order optimality conditions have been provided.

After the inspection of some differentiability properties of the state solution operator, a FEM discretization of the problem has been introduced. For this discretization, a priori error estimates have been derived, showing a quadratic convergence rate. To our best knowledge, this is the first result about convergence rates obtained for the discretization of two-dimensional Stokes problem in a shape optimization context. Numerical tests have been performed to assess the validity of the theoretical results.

The present paper aims at representing a first step towards the application of the reference domain approach to Navier-Stokes equations (two-dimensional or even three-dimensional), with the derivation of suitable convergence rates for the FEM discretization errors. Such a goal will require a more general analytical framework, and comprehensive results like those presented in [33] will be very useful.

\section{Appendix A. Additional Regularity}

In this Appendix we want to show a possible way to derive the regularity properties stated in Assumption 3.16, starting from suitable requests on data and a regularity result on Stokes problem with mixed boundary conditions.

At first, let us state a preliminary result about the transformation of norms defined on the reference domain $\left(\Omega_{0}\right)$ and on the physical one $\left(\Omega_{q}\right)$.

Lemma A.1. Let $k \in \mathbb{N}$ be fixed, $\varphi \in H^{k}\left(\Omega_{0}\right)$ and $q \in W^{k, \infty}(I)$. It holds that

$$
\varphi \circ T_{q}^{-1} \in H^{k}\left(\Omega_{q}\right), \quad c_{1}\|q\|_{W^{k, \infty}(I)}\left\|\varphi \circ T_{q}^{-1}\right\|_{H^{k}\left(\Omega_{q}\right)} \leq\|\varphi\|_{H^{k}\left(\Omega_{0}\right)} \leq c_{2}\|q\|_{W^{k, \infty}(I)}\left\|\varphi \circ T_{q}^{-1}\right\|_{H^{k}\left(\Omega_{q}\right)} .
$$

Vice versa, it holds that $\widetilde{\varphi} \in H^{k}\left(\Omega_{q}\right)$ implies $\widetilde{\varphi} \circ T_{q} \in H^{k}\left(\Omega_{0}\right)$, together with similar inequalities.

In connection with this lemma, we restrict a little the set of admissible controls. From now on, the definition of $Q^{a d}$ will contain also the belonging of control functions $q$ to $W^{3, \infty}(I)$ and the existence of a constant $c_{\infty}>0$ such that

$$
\|q\|_{W^{3, \infty}} \leq c_{\infty} \quad \forall q \in Q^{a d}
$$

that is

$$
Q^{a d}:=\left\{q \in W^{3, \infty}(I) \cap H_{0}^{1}(I): q(x) \leq 1-\varepsilon, \forall x \in I, \text { and }\|q\|_{W^{3, \infty}(I)} \leq c_{\infty}\right\} .
$$

Thanks to the above definition and to Lemma A.1, when handling functions belonging to $H^{k}\left(\Omega_{0}\right)$ or $H^{k}\left(\Omega_{q}\right)$ for $k \leq 3$, we can indifferently consider their norm in the physical domain $\Omega_{q}$ or in the reference domain $\Omega_{0}$.

The last ingredient that we need in order to prove a regularity result for the solution of our transformed problem (2.8) is represented by additional regularity requests on data. Since we want regularity not only for the solution of (2.8), but also for its derivatives w.r.t. the control, namely $S^{\prime}(q)(\delta q), S^{\prime \prime}(q)(\delta q, \delta q)$, we have to assume a slightly stronger regularity of data than that considered in Assumption 3.7.

Assumption A.2. Data functions have the following regularity:

$$
\nu \in H^{5}(\widehat{\Omega}), \quad \eta \in H^{4}(\widehat{\Omega}), \quad \mathbf{f} \in\left[H^{4}(\widehat{\Omega})\right]^{2}, \quad \mathbf{g}_{D} \in\left[H^{5 / 2}\left(\Gamma_{3}\right)\right]^{2}, \quad \mathbf{g}_{N} \in\left[H^{3 / 2}\left(\Gamma_{1}\right)\right]^{2},
$$

and suitable compatibility conditions hold on data.

We are now ready to state a regularity result for the state variables and their shape derivatives, justifying Assumption 3.16. 
Theorem A.3. Under Assumptions 3.7, A.2, there exist three positive constants $c_{0}, c_{1}, c_{2}$, such that for any $q \in Q^{a d}$ and for any $\delta q, \tau q \in \delta Q$, and independently from them, it holds that

$$
S(q), S^{\prime}(q)(\delta q), S^{\prime \prime}(q)(\delta q, \tau q) \in\left[H^{3}\left(\Omega_{0}\right)\right]^{2} \times H^{2}\left(\Omega_{0}\right) \quad \text { and }
$$

(a) $\|S(q)\|_{\left[H^{3}\left(\Omega_{0}\right)\right]^{2} \times H^{2}\left(\Omega_{0}\right)} \leq c_{0}$

(b) $\left\|S^{\prime}(q)(\delta q)\right\|_{\left[H^{3}\left(\Omega_{0}\right)\right]^{2} \times H^{2}\left(\Omega_{0}\right)} \leq c_{1}\|\delta q\|_{H^{3}(I)}$

(c) $\left\|S^{\prime \prime}(q)(\delta q, \tau q)\right\|_{\left[H^{3}\left(\Omega_{0}\right)\right]^{2} \times H^{2}\left(\Omega_{0}\right)} \leq c_{2}\|\delta q\|_{H^{3}(I)}\|\tau q\|_{H^{3}(I)}$.

Proof. Let $q \in Q^{a d}$, consider solution $(\mathbf{u}, p)=S(q)$ of the transformed problem (2.8) and remind that its physical counterpart $(\widetilde{\mathbf{u}}, \widetilde{p})=\widetilde{S}(q)$ is the solution of Stokes problem (2.4) on $\Omega_{q}$.

Now, we can verify the hypotheses of Assumption 3.7: $\Omega_{q}$ is surely an open bounded subset of $\mathbb{R}^{2}$; its boundary $\Gamma_{q}$ is $C^{1,1}$ because it is the graph of the control function $q \in Q^{a d} \subset H^{3}(I) \subset C^{1,1}\left(\bar{\Omega}_{q}\right)$ and for the same reason its terminal points cannot present a concave angle; the regularity of external force and boundary data, together with the compatibility conditions, are given by Assumption A.2. Then, Assumption 3.7 holds and we have $(\widetilde{\mathbf{u}}, \widetilde{p}) \in\left[H^{3}\left(\Omega_{q}\right)\right]^{2} \times H^{2}\left(\Omega_{q}\right)$ and $\|\widetilde{\mathbf{u}}\|_{\left[H^{3}\left(\Omega_{q}\right)\right]^{2}}+\|\widetilde{p}\|_{H^{2}\left(\Omega_{q}\right)} \leq c\left(\mathbf{f}, \mathbf{g}_{D}, \mathbf{g}_{N}, \widehat{\Omega}\right)$. Finally, the results on $(\widetilde{\mathbf{u}}, \widetilde{p})$ directly transfer to $(\mathbf{u}, p)$, thanks to Lemma A.1.

For points (b) and (c), the proof is exactly the same, considering Assumption A.2 in order to control the more complex right-hand sides appearing dealing with $S^{\prime}(q)(\delta q)$ and $S^{\prime \prime}(q)(\delta q, \tau q)$, with the aim of proving the validity of the hypotheses of Assumption 3.7. The dependence on $\|\delta q\|_{H^{3}(I)},\|\tau q\|_{H^{3}(I)}$ of the right-hand side of the inequalities comes out from the bounds on the coefficients, similar to those reported in Proposition 3.4.

So far we have obtained a regularity result for the state variables: now we want to show that the optimal control belongs to $H^{5}(I)$. Indeed, this regularity holds for any $q \in Q^{\text {ad }}$ satisfying the first order optimality condition, as stated in the following result:

Theorem A.4. Let $\bar{q} \in Q^{a d}$ be such that optimality condition (4.1) holds in $\bar{q}$. Then it holds that $\bar{q} \in H^{5}(I)$.

Proof. Let us take into account Hadamard formula for $j^{\prime}$, given by Lemma 4.1, i.e. $j^{\prime}(q)(\delta q)=\alpha\left(q^{\prime \prime}, \delta q^{\prime \prime}\right)_{I}+$ $(\Psi, \delta q)_{I}$, with $\Psi=\Psi(q)$ defined in (4.9). We start by showing that $\Psi \in H^{1}(I)$. We preliminary notice that $\Psi_{1}(q):=\beta\left(\int_{I} q(x) \mathrm{d} x-\bar{V}\right)$ is constant, then it certainly belongs to $H^{1}(I)$.

Let us now discuss $\Psi_{2}(q)$ (see (4.6) for proper definition). The regularity Theorem A.3 can be applied to both the state velocity $\mathbf{u}$ and the adjoint velocity z. Then, thanks to the definition (A.1) and Lemma A.1, we get

$$
(\widetilde{\mathbf{u}}, \widetilde{\mathbf{z}})=(\mathbf{u}, \mathbf{z}) \circ T_{q} \quad \in\left[H^{3}\left(\Omega_{q}\right)\right]^{2} \times\left[H^{3}\left(\Omega_{q}\right)\right]^{2} .
$$

Taking the traces of $\nabla \widetilde{\mathbf{u}}, \nabla \widetilde{\mathbf{z}}$ on the boundary $\Gamma_{q}$ and using its parametrization $\gamma: x \mapsto(x, q(x))$, we get

$$
(\nabla \widetilde{\mathbf{u}}, \nabla \widetilde{\mathbf{z}}) \quad \in \quad\left[H^{3 / 2}(I)\right]^{2 \times 2} \times\left[H^{3 / 2}(I)\right]^{2 \times 2} .
$$

Thanks to this regularity, together with the continuous embedding $H^{3 / 2}(I) \hookrightarrow W^{1,4}(I)$, we can conclude that $\Psi_{2}(q)$ belongs to $H^{1}(I)$. Hence, $\Psi(q) \in H^{1}(I)$.

Now, taking $\bar{q} \in Q^{a d}$ such that the optimality condition $j^{\prime}(\bar{q})(\delta q)=0$ holds, we get

$$
\int_{I} \bar{q}^{\prime \prime} \delta q^{\prime \prime} \mathrm{d} x=-\int_{I} \frac{1}{\alpha} \Psi \delta q \mathrm{~d} x \quad \forall \delta q \in C_{0}^{\infty}(\bar{I}) .
$$

Finally, we observe that (A.2) is equivalent to say that the fourth weak derivative of $\bar{q}$ is exactly $-\frac{1}{\alpha} \Psi$. Being $\alpha$ a non-zero constant and belonging $\Psi$ to $H^{1}(I)$, we get $\bar{q}^{(i v)} \in H^{1}(I)$. Since we already have $\bar{q} \in W^{3, \infty} \subset H^{3}(I)$ (see (A.1)), we obtain the thesis, i.e. $\bar{q} \in H^{5}(I)$. 


\section{Appendix B. Results for the Coercivity of Functional $j$}

In this appendix, we present two useful results for the proof of Lemma 3.14. The first concerns the sequential continuity of the state operator derivatives w.r.t. the variations of control.

Lemma B.1. Let $q \in Q^{a d}$ and consider a sequence $\left\{\delta q_{n}\right\}_{n \in \mathbb{N}} \subset \delta Q$. If there exists a $\delta q \in \delta Q$ such that $\delta q_{n} \rightarrow \delta q$ in $C^{1}(\bar{I})$, then

(a) $S^{\prime}(q)\left(\delta q_{n}\right) \rightarrow S^{\prime}(q)(\delta q)$ in $V \times P$

(b) $S^{\prime \prime}(q)\left(\delta q_{n}, \delta q_{n}\right) \rightarrow S^{\prime \prime}(q)(\delta q, \delta q)$ in $V \times P$

Proof. Because of the linearity and the well-posedness of problems (3.2), (3.3), it suffices to prove the convergence of the right-hand sides in $V^{\prime} \times P^{\prime}$ : this is obtained from the continuity of $\dot{F}, \dot{a}, \dot{b}, \ddot{F}, \ddot{a}, \ddot{b}$ w.r.t. the variation $\delta q$.

We just give an example of the steps to be taken, processing a term from $\ddot{a}(q, \delta q, \delta q)(\mathbf{u}, \mathbf{v})$ :

$$
\begin{aligned}
& \left|\left(\nabla \nu^{q} \cdot \mathscr{T}_{\delta q_{n}} \nabla \mathbf{u} A_{q, \delta q_{n}}^{\prime}, \nabla \mathbf{v}\right)-\left(\nabla \nu^{q} \cdot \mathscr{T}_{\delta q} \nabla \mathbf{u} A_{q, \delta q}^{\prime}, \nabla \mathbf{v}\right)\right| \\
& \leq\left|\left(\nabla \nu^{q} \cdot \mathscr{T}_{\delta q_{n}} \nabla \mathbf{u}\left(A_{q, \delta q_{n}}^{\prime}-A_{q, \delta q}^{\prime}\right), \nabla \mathbf{v}\right)\right|+\left|\left(\nabla \nu^{q} \cdot\left(\mathscr{T}_{\delta q_{n}}-\mathscr{T}_{\delta q}\right) \nabla \mathbf{u} A_{q, \delta q}^{\prime}, \nabla \mathbf{v}\right)\right| \\
& \leq\|\nu\|_{W^{1, \infty}(\widehat{\Omega})}\|\nabla \mathbf{u}\|\|\nabla \mathbf{v}\|\left(\left\|A_{q, \delta q_{n}}^{\prime}-A_{q, \delta q}^{\prime}\right\|_{\infty}\left\|\mathscr{T}_{\delta q_{n}}\right\|_{\infty}+\left\|\mathscr{T}_{\delta q_{n}}-\mathscr{T}_{\delta q}\right\|_{\infty}\left\|A_{q, \delta q}^{\prime}\right\|_{\infty}\right) .
\end{aligned}
$$

The convergence of $\delta q_{n}$ in $C^{1}(\bar{I})$ implies the uniform convergence of $A_{q, \delta q_{n}}^{\prime}-A_{q, \delta q}^{\prime}$ and $\mathscr{T}_{\delta q_{n}}-\mathscr{T}_{\delta q}$ to zero, as it can be seen from the definition of such quantities. Moreover, being $\left\{\delta q_{n}\right\}$ bounded in $C^{1}(\bar{I})$, Proposition 3.4 ensures that $\left\|\mathscr{T}_{\delta q_{n}}\right\|_{\infty},\left\|A_{q, \delta q}^{\prime}\right\|_{\infty}$ are bounded themselves.

From Lemma B.1, using the Dominated Convergence Theorem and the compact embedding $H^{2}(I) \subset \subset C^{1}(\bar{I})$ yields a similar result for the derivatives of the cost functional $j$ :

Corollary B.2. Let $q \in Q^{a d}$ and $\left\{\delta q_{n}\right\}_{n \in \mathbb{N}} \subset \delta Q$ such that there exists a $\delta q \in \delta Q$ for which $\delta q_{n} \rightarrow \delta q$ in $H^{2}(I)$. Then,

$$
j^{\prime}(q)\left(\delta q_{n}\right) \underset{n \rightarrow \infty}{\longrightarrow} j(q)(\delta q), \quad j^{\prime \prime}(q)(\delta q, \delta q) \leq \liminf _{n \rightarrow \infty} j^{\prime \prime}(q)\left(\delta q_{n}, \delta q_{n}\right) .
$$

\section{REFERENCES}

[1] G. Allaire, Conception optimale de structures. Springer-Verlag (2007).

[2] P.F. Antonietti, A. Borzì and M. Verani, Multigrid shape optimization governed by elliptic PDEs. SIAM J. Control Optim. 51 (2013) 1417-1440.

[3] F. Ballarin, A. Manzoni, G. Rozza and S. Salsa, Shape Optimization by Free-Form Deformation: Existence Results and Numerical Solution for Stokes Flows. J. Sci. Comput. 60 (2013) 537-563.

[4] D. Begis and R. Glowinski, Application de la méthode des éléments finis à l'approximation d'un problème de domaine optimal. Méthodes de résolution des problèmes approchés. Appl. Math. Optim. 2 (1975) 130-169.

[5] F. Bélahcène and J.A. Desideri, Paramétrisation de Bézier adaptative pour l'optimisation de forme en Aérodynamique. Research Report RR-4943, INRIA (2003).

[6] J.A. Bello, E. Fernández-Cara, J. Lemoine and J. Simon, The Differentiability of the Drag with Respect to the Variations of a Lipschitz Domain in a Navier-Stokes Flow. SIAM J. Control Optim. 35 (1997) 626-640.

[7] S.C. Brenner and R. Scott, The mathematical theory of finite element methods. Springer Texts Appl. Math., 3rd edition (2008).

[8] F. Brezzi, On the existence uniqueness and approximation of saddle point problems arising from lagrangian multipliers. Rev. Fr. Automat. Infor. 8 (1974) 129-151.

[9] D. Chenais and E. Zuazua, Controllability of an elliptic equation and its finite difference approximation by the shape of the domain. Numer. Math. 95 (2003) 63-99.

[10] D. Chenais and E. Zuazua, Finite-element approximation of 2D elliptic optimal design. J. Math. Pure Appl. 85 (2006) $225-249$.

[11] F. de Gournay, G. Allaire and F. Jouve, Shape and topology optimization of the robust compliance via the level set method. ESAIM: COCV 14 (2008) 43-70. 
[12] M.C. Delfour and J.P. Zolésio, Shapes and geometries. Society for Industrial and Applied Mathematics (SIAM), 2nd edition (2011).

[13] G. Dogan, P. Morin, R.H. Nochetto and M. Verani, Discrete gradient flows for shape optimization and applications. Comput. Methods Appl. Mech. 196 (2007) 3898-3914.

[14] R.G. Durán, An elementary proof of the continuity from $L_{0}^{2}(\Omega)$ to $H_{0}^{1}(\Omega)^{n}$ of Bogovskii's right inverse of the divergence. Rev. Un. Mat. Argentina 53 (2013) 59-78.

[15] K. Eppler, Second derivatives and sufficient optimality conditions for shape functionals. Control Cybern. 29 (2000) 485-511.

[16] K. Eppler, H. Harbrecht and R. Schneider, On Convergence in Elliptic Shape Optimization. SIAM J. Control Optim. 46 (2007) 61-83.

[17] I. Fumagalli, Shape optimization for Stokes flows: a reference-domain approach. M.Sc. thesis, Politecnico di Milano, Italy (2013). Available at http://www mate.polimi.it/biblioteca/?pp=view\&id=537\&collezione=tesi\&L=i.

[18] V. Girault and P.A. Raviart, Finite element methods for Navier-Stokes equations. Springer-Verlag (1986).

[19] M.D. Gunzburger, Perspectives in flow control and optimization. Society for Industrial and Applied Mathematics (SIAM) (2003).

[20] M.D. Gunzburger, H. Kim and S. Manservisi, On a shape control problem for the stationary Navier-Stokes equations. ESAIM: M2AN 34 (2000) 1233-1258.

[21] B. Guo and C. Schwab, Analytic regularity of Stokes flow on polygonal domains in countably weighted Sobolev spaces. J. Comput. Appl. Math. 190 (2006) 487-519.

[22] J. Haslinger and R.A.E. Mäkinen, Introduction to shape optimization. Society for Industrial and Applied Mathematics (SIAM) (2003).

[23] J. Haslinger and P. Neittaanmäki, Finite element approximation for optimal shape design: Theory and applications. Wiley Chichester (1988).

[24] K. Ito and K. Kunisch, Lagrange multiplier approach to variational problems and applications. Society for Industrial and Applied Mathematics (SIAM) (2008).

[25] A.M. Khludnev and J. Sokolowski, Modelling and control in solid mechanics. Birkhäuser Verlag, Basel (1997).

[26] B. Kiniger and B. Vexler, A priori error estimates for finite element discretizations of a shape optimization problem. ESAIM: M2AN 47 (2013) 1733-1763.

[27] M. Laumen, Newton's method for a class of optimal shape design problems. SIAM J. Optim. 10 (2000) $503-533$.

[28] J.L. Lions, Optimal control of systems governed by partial differential equations. Springer-Verlag (1971).

[29] A. Logg, K.A. Mardal and G.N. Wells, Automated Solution of Differential Equations by the Finite Element Method. Springer (2012).

[30] P. Morin, R.H. Nochetto, M.S. Pauletti and M. Verani, Adaptive finite element method for shape optimization. ESAIM: COCV 18 (2012) 1122-1149.

[31] J. Nocedal and S.J. Wright, Numerical optimization. 2nd edition, Springer (2006).

[32] O. Pironneau, On optimum profiles in Stokes flow. J. Fluid Mech. 59 (1973) 117-128.

[33] P. Plotnikov and J. Sokołowski, Compressible Navier-Stokes equations. Theory and shape optimization. Birkhäuser/Springer, Basel (2012).

[34] A. Quarteroni and A. Valli, Numerical Approximation of Partial Differential Equations. Series Comput. Math. Springer (2008).

[35] J. Sokolowsky and J.P. Zolésio, Introduction to Shape Optimization. Springer-Verlag (1992). 